KYUNGPOOK Math. J. 52(2012), 109-136

http://dx.doi.org/10.5666/KMJ.2012.52.2.109

\title{
Serendipitous Functional Relations Deducible from Certain Generalized Triple Hypergeometric Functions
}

\author{
Junesang Choi*, Anvar Hasanov and Mamasali Turaev \\ Department of Mathematics, Dongguk University, Gyeongju, 780-714, Korea \\ e-mail: junesang@mail.dongguk.ac.kr, ahasanov@dongguk.ac.kr \\ and mturaev@dongguk.ac.kr
}

ABstract. We aim at presenting certain unexpected functional relations among various hypergeometric functions of one or several variables (for example, see the identities in Corollary 5) by making use of Carlson's method employed in his work (Some extensions of Lardner's relations between ${ }_{0} F_{3}$ and Bessel functions, SIAM J. Math. Anal. 1(2)(1970), $232-242)$.

\section{Introduction}

Investigation of multiple hypergeometric functions is essentially motivated by the fact that the solutions of many applied problems involving the thermal conductivity and dynamics, electromagnetic oscillation and aerodynamics, quantum mechanics and potential theory are obtainable with the help of such hypergeometric (higher and special or transcendent) functions (see [7, 11, 25, 27]). Such functions are often referred to as special functions in mathematical physics. They are mainly appeared in the solution of partial differential equations by using harmonic analysis method [9]. In view of various applications, it is important as well as interesting in itself to conduct a continuous research on multiple hypergeometric functions. In fact, in Srivastava and Karlsson's work [33], there is an extensive list of as many as 205 hypergeometric functions of three variables together with their region of convergence. It is noted that Riemann's functions and the fundamental solutions of the degenerate second-order partial differential equations are expressible by using hypergeometric functions of several variables (see $[2,4,5,6,12,13,14,15,16,17,26,29,36,37,38]$ ). For solutions of the boundary-value problems for the involved partial differential equations, we need to investigate certain properties of hypergeometric functions of several variables (see $[18,19,20,21,22,28,34])$.

\footnotetext{
* Corresponding Author.

Received January 12, 2011; accepted November 24, 2011.

2010 Mathematics Subject Classification: Primary 33C20; Secondary 44A45.

Key words and phrases: eneralized hypergeometric series, Pochhammer symbol, Gauss function, Appell-Kampé de Fériet functions, Bessel functions, Kelvin's functions, Srivastava's hypergeometric functions.
} 
Lardner [23] gave some connections between Bessel functions and hypergeometric ${ }_{0} F_{3}$-series, for example,

$$
{ }_{0} F_{3}\left(\frac{1}{2}, \frac{1}{2}, 1 ; z\right)=\frac{1}{2}\left[J_{0}\left(4 z^{\frac{1}{4}}\right)+I_{0}\left(4 z^{\frac{1}{4}}\right)\right]
$$

and

(1.2) ber $(x)={ }_{0} F_{3}\left(\frac{1}{2}, \frac{1}{2}, 1 ;-\frac{x^{4}}{256}\right)$, and $\operatorname{bei}(x)=\frac{x^{2}}{4}{ }_{0} F_{3}\left(\frac{3}{2}, \frac{3}{2}, 1 ;-\frac{x^{4}}{256}\right)$,

where $J_{\nu}$ and $I_{\nu}$ denote a Bessel function and a modified Bessel function of order $\nu$ (see [1]; also [35]) defined by

$$
\begin{aligned}
& J_{\nu}(z)=\frac{\left(\frac{z}{2}\right)^{\nu}}{\Gamma(\nu+1)}{ }_{0} F_{1}\left(-; \nu+1 ;-\frac{z^{2}}{4}\right) \\
& \text { and } I_{\nu}(z)=\frac{\left(\frac{z}{2}\right)^{\nu}}{\Gamma(\nu+1)}{ }_{0} F_{1}\left(-; \nu+1 ; \frac{z^{2}}{4}\right),
\end{aligned}
$$

and $\operatorname{ber}(x)$ and bei $(x)$ ( $x$ real) denote the Kelvin's functions (see [10, p. 6]) defined by

$$
\operatorname{ber}(x)+i \operatorname{bei}(x)=J_{0}\left(x e^{i \frac{3}{4} \pi}\right)=I_{0}\left(x e^{i \frac{1}{4} \pi}\right) .
$$

Carlson [8] generalized these results for arbitrary parameters to give the following results

$$
{ }_{0} F_{3}\left(\frac{1}{2}, c, c+\frac{1}{2} ; z\right)=\frac{1}{2} \Gamma(2 c)\left(2 z^{\frac{1}{4}}\right)^{1-2 c}\left[I_{2 c-1}\left(4 z^{\frac{1}{4}}\right)+J_{2 c-1}\left(4 z^{\frac{1}{4}}\right)\right]
$$

and

(1.6) ${ }_{0} F_{3}\left(\frac{3}{2}, c, c+\frac{1}{2} ; z\right)=\frac{1}{2} \Gamma(2 c)\left(2 z^{\frac{1}{4}}\right)^{-2 c}\left[I_{2 c-2}\left(4 z^{\frac{1}{4}}\right)-J_{2 c-2}\left(4 z^{\frac{1}{4}}\right)\right]$.

Srivastava $[30,31,33]$ discovered the existence of three additional complete triple hypergeometric functions $H_{A}, H_{B}$ and $H_{C}$ of the second order. One of them is presented as follows:

$$
H_{A}\left(a_{1}, a_{2}, a_{3} ; c_{1}, c_{2} ; x, y, z\right)=\sum_{m, n, p=0}^{\infty} \frac{\left(a_{1}\right)_{m+p}\left(a_{2}\right)_{m+n}\left(a_{3}\right)_{n+p}}{\left(c_{1}\right)_{m}\left(c_{2}\right)_{n+p} m ! n ! p !} x^{m} y^{n} z^{p}
$$

where $\mathbb{C}$ and $\mathbb{Z}_{0}^{-}$denote the set of complex numbers and the set of nonpositive integers, respectively, and $(\lambda)_{n}$ is the Pochhammer symbol defined (for $\lambda \in \mathbb{C}$ ) by (see [32]):

$$
\begin{array}{rlr}
(\lambda)_{n} & :=\left\{\begin{array}{cl}
1 & (n=0) \\
\lambda(\lambda+1) \cdots \lambda(\lambda+n-1) & (n \in \mathbb{N}:=\{1,2,3, \ldots\})
\end{array}\right. \\
& =\frac{\Gamma(\lambda+n)}{\Gamma(\lambda)} \quad\left(\lambda \in \mathbb{C} \backslash \mathbb{Z}_{0}^{-}\right),
\end{array}
$$


$\Gamma(x)$ is well-known Gamma function. The three-dimensional region of convergence of (1.7) is given by Srivastava and Karlsson [33]: $(|x|:=r<1,|y|:=s<1,|z|:=$ $t<(1-r)(1-s))$, where the positive quantities $r, s$ and $t$ are associated with radii of convergence.

Here, by simply splitting Srivastava's hypergeometric function $H_{A}$ into eight parts, we show how some useful and generalized relations between Srivastava's hypergeometric functions $H_{A}$ and $F^{(3)}$ can be obtained. As a particular case, some decomposition formulas for the generalized Srivastava's hypergeometric function $F^{(3)}$ were obtained by means of Gauss's hypergeometric function and vice-versa. The other main results are shown to be specialized to yield certain relations between functions $\Phi_{1}$ and $\Psi_{1},{ }_{0} F_{1},{ }_{1} F_{1},{ }_{0} F_{3}, F_{2: 0 ; 0}^{2: 1 ; 1}$. Some other interesting functional relations between the exponential function, the hyperbolic functions, and modified Bessel functions are considered as well.

\section{Relationships between Srivastava's hypergeometric functions $H_{A}$ and $F^{(3)}$}

In this section we establish some interesting and useful identities associated with Srivastava's functions $H_{A}$ and $F^{(3)}$. For this purpose we simply separate the summations in (1.7) into odd and even powers of each of $x^{m}, y^{n}$, and $z^{p}$. In fact, for any complex $c_{1}, c_{2} \in \mathbb{C} \backslash \mathbb{Z}_{0}^{-}$, and any finite complex $x, y$, and $z$, the series $H_{A}$ converges absolutely in the region of convergence and can therefore be rearranged as in the following eight summations:

$$
\begin{aligned}
& H_{A}\left(a_{1}, a_{2}, a_{3} ; c_{1}, c_{2} ; x, y, z\right) \\
= & \sum_{i, j, k=0}^{\infty} \frac{\left(a_{1}\right)_{2(i+k)}\left(a_{2}\right)_{2(i+j)}\left(a_{3}\right)_{2(j+k)}}{\left(c_{1}\right)_{2 i}\left(c_{2}\right)_{2(j+k)}(2 i) !(2 j) !(2 k) !} x^{2 i} y^{2 j} z^{2 k} \\
& +x \sum_{i, j, k=0}^{\infty} \frac{\left(a_{1}\right)_{2(i+k)+1}\left(a_{2}\right)_{2(i+j)+1}\left(a_{3}\right)_{2(j+k)}}{\left(c_{1}\right)_{2 i+1}\left(c_{2}\right)_{2(j+k)}(2 i+1) !(2 j) !(2 k) !} x^{2 i} y^{2 j} z^{2 k} \\
& +y \sum_{i, j, k=0}^{\infty} \frac{\left(a_{1}\right)_{2(i+k)}\left(a_{2}\right)_{2(i+j)+1}\left(a_{3}\right)_{2(j+k)+1}}{\left(c_{1}\right)_{2 i}\left(c_{2}\right)_{2(j+k)+1}(2 i) !(2 j+1) !(2 k) !} x^{2 i} y^{2 j} z^{2 k} \\
& +z \sum_{i, j, k=0}^{\infty} \frac{\left(a_{1}\right)_{2(i+k)+1}\left(a_{2}\right)_{2(i+j)}\left(a_{3}\right)_{2(j+k)+1}}{\left(c_{1}\right)_{2 i}\left(c_{2}\right)_{2(j+k)+1}(2 i) !(2 j) !(2 k+1) !} x^{2 i} y^{2 j} z^{2 k} \\
& +x y \sum_{i, j, k=0}^{\infty} \frac{\left(a_{1}\right)_{2(i+k)+1}\left(a_{2}\right)_{2(i+j)+2}\left(a_{3}\right)_{2(j+k)+1}}{\left(c_{1}\right)_{2 i+1}\left(c_{2}\right)_{2(j+k)+1}(2 i+1) !(2 j+1) !(2 k) !} x^{2 i} y^{2 j} z^{2 k} \\
& +x z \sum_{i, j, k=0}^{\infty} \frac{\left(a_{1}\right)_{2(i+k)+2}\left(a_{2}\right)_{2(i+j)+1}\left(a_{3}\right)_{2(j+k)+1}}{\left(c_{1}\right)_{2 i+1}\left(c_{2}\right)_{2(j+k)+1}(2 i+1) !(2 j) !(2 k+1) !} x^{2 i} y^{2 j} z^{2 k}
\end{aligned}
$$




$$
\begin{aligned}
& +y z \sum_{i, j, k=0}^{\infty} \frac{\left(a_{1}\right)_{2(i+k)+1}\left(a_{2}\right)_{2(i+j)+1}\left(a_{3}\right)_{2(j+k)+2}}{\left(c_{1}\right)_{2 i}\left(c_{2}\right)_{2(j+k)+2}(2 i) !(2 j+1) !(2 k+1) !} x^{2 i} y^{2 j} z^{2 k} \\
& +x y z \sum_{i, j, k=0}^{\infty} \frac{\left(a_{1}\right)_{2(i+k)+2}\left(a_{2}\right)_{2(i+j)+2}\left(a_{3}\right)_{2(j+k)+2}}{\left(c_{1}\right)_{2 i+1}\left(c_{2}\right)_{2(j+k)+2}(2 i+1) !(2 j+1) !(2 k+1) !} x^{2 i} y^{2 j} z^{2 k} .
\end{aligned}
$$

Now making use of the following well-known (or easily derivable) identity for the Pochhammer symbol (see [23, 24]):

$$
(\alpha)_{2 m}=2^{2 m}\left(\frac{\alpha}{2}\right)_{m}\left(\frac{\alpha}{2}+\frac{1}{2}\right)_{m} \quad\left(m \in \mathbb{N}_{0}:=\mathbb{N} \cup\{0\}\right),
$$

after some simplification, we obtain

Theorem 1. The following relationship between $H_{A}$ and $F^{(3)}$ holds true.

$$
H_{A}\left(a_{1}, a_{2}, a_{3} ; c_{1}, c_{2} ; x, y, z\right)
$$

$$
\begin{aligned}
& =F^{(3)}\left[\begin{array}{rrrrrrrr}
-:: & \frac{a_{2}}{2}, \frac{a_{2}+1}{2} ; & \frac{a_{3}}{2}, \frac{a_{3}+1}{2} ; & \frac{a_{1}}{2}, \frac{a_{1}+1}{2} ; & -1 & -; & -; & x^{2}, y^{2}, z^{2} \\
-:: & -; & \frac{c_{2}}{2}, \frac{c_{2}+1}{2} ; & -; & \frac{c_{1}+1}{2}, \frac{1}{2} ; & \frac{1}{2} ; & \frac{1}{2} ;
\end{array}\right. \\
& +\frac{a_{1} a_{2}}{c_{1}} x F^{(3)}\left[\begin{array}{lrrrr}
-:: & \frac{a_{2}+1}{2}, & \frac{a_{2}+2}{2} ; & \frac{a_{3}}{2}, \frac{a_{3}+1}{2} ; & \frac{a_{1}}{2}, \frac{a_{1}+1}{2} ; \\
-:: & -; & \frac{c_{2}}{2}, \frac{c_{2}+1}{2} ; & -;
\end{array}\right. \\
& \left.\frac{c_{1}+1}{2}, \frac{c_{1}+2}{2}, \frac{3}{2} ; \quad-\frac{1}{2} ; \quad-\frac{1}{2} ; x^{2}, y^{2}, z^{2}\right] \\
& +\frac{a_{2} a_{3}}{c_{2}} y F^{(3)}\left[\begin{array}{rrrr}
-:: & \frac{a_{2}+1}{2}, \frac{a_{2}+2}{2} ; & \frac{a_{3}+1}{2}, \frac{a_{3}+2}{2} ; & \frac{a_{1}}{2}, \frac{a_{1}+1}{2} ; \\
-:: & -; & \frac{c_{2}+1}{2}, & \frac{c_{2}+2}{2} ;
\end{array}\right. \\
& \left.\frac{c_{1}}{2}, \frac{c_{1}+1}{2}, \frac{1}{2} ; \quad-\frac{3}{2} ; \quad-\frac{1}{2} ; x^{2}, y^{2}, z^{2}\right] \\
& +\frac{a_{1} a_{3}}{c_{2}} z F^{(3)}\left[\begin{array}{rrrr}
-:: & \frac{a_{2}}{2}, \frac{a_{2}+1}{2} ; & \frac{a_{3}+1}{2}, \frac{a_{3}+2}{2} ; & \frac{a_{1}+1}{2}, \frac{a_{1}+2}{2} ; \\
-:: & -; & \frac{c_{2}+1}{2}, \frac{c_{2}+2}{2} ; & -;
\end{array}\right. \\
& \left.\frac{c_{1}}{2}, \frac{c_{1}+1}{2}, \frac{1}{2} ; \quad \frac{1}{2} ; \quad \frac{3}{2} ; x^{2}, y^{2}, z^{2}\right] \\
& +\frac{a_{1} a_{2}\left(a_{2}+1\right) a_{3}}{c_{1} c_{2}} x y F^{(3)}\left[\begin{array}{rrrrr}
-:: & \frac{a_{2}+2}{2}, & \frac{a_{2}+3}{2} ; & \frac{a_{3}+1}{2}, \frac{a_{3}+2}{2} ; & \frac{a_{1}+1}{2}, \frac{a_{1}+2}{2} ; \\
-:: & -; & \frac{c_{2}+1}{2}, \frac{c_{2}+2}{2} ; & -;
\end{array}\right. \\
& \left.\frac{c_{1}+1}{2}, \frac{c_{1}+2}{2}, \frac{-3}{2} ; \quad-\frac{3}{2} ; \quad-\frac{1}{2} ; x^{2}, y^{2}, z^{2}\right]
\end{aligned}
$$




$$
\begin{aligned}
& +\frac{a_{1}\left(a_{1}+1\right) a_{2} a_{3}}{c_{1} c_{2}} x z F^{(3)}\left[\begin{array}{rrrrr}
-:: & \frac{a_{2}+1}{2}, & \frac{a_{2}+2}{2} ; & \frac{a_{3}+1}{2}, \frac{a_{3}+2}{2} ; & \frac{a_{1}+2}{2}, \frac{a_{1}+3}{2} ; \\
-:: & -; & \frac{c_{2}+1}{2}, & \frac{c_{2}+2}{2} ; & -;
\end{array}\right. \\
& \left.\frac{c_{1}+1}{2}, \frac{c_{1}+2}{2}, \frac{-3}{2} ; \quad \frac{1}{2} ; \quad \frac{3}{2} ; x^{2}, y^{2}, z^{2}\right] \\
& +\frac{a_{1} a_{2} a_{3}\left(a_{3}+1\right)}{c_{2}\left(c_{2}+1\right)} y z F^{(3)}\left[\begin{array}{rrrrr}
-:: & \frac{a_{2}+1}{2}, \frac{a_{2}+2}{2} ; & \frac{a_{3}+2}{2}, \frac{a_{3}+3}{2} ; & \frac{a_{1}+1}{2}, \frac{a_{1}+2}{2} ; \\
-:: & -; & \frac{c_{2}+2}{2}, \frac{c_{2}+3}{2} ; & -;
\end{array}\right. \\
& \left.\frac{c_{1}}{2}, \frac{c_{1}+1}{2}, \frac{1}{2} ; \quad-\frac{3}{2} ; \quad \frac{3}{2} ; x^{2}, y^{2}, z^{2}\right] \\
& +\frac{a_{1}\left(a_{1}+1\right) a_{2}\left(a_{2}+1\right) a_{3}\left(a_{3}+1\right)}{c_{1} c_{2}\left(c_{2}+1\right)} x y z \\
& \cdot F^{(3)}\left[\begin{array}{rrrr}
-:: & \frac{a_{2}+2}{2}, \frac{a_{2}+3}{2} ; & \frac{a_{3}+2}{2}, \frac{a_{3}+3}{2} ; & \frac{a_{1}+2}{2}, \frac{a_{1}+3}{2} ; \\
-:: & -; & \frac{c_{2}+2}{2}, \frac{c_{2}+3}{2} ; & -;
\end{array}\right. \\
& \left.\frac{c_{1}+1}{2}, \frac{c_{1}+2}{2}, \frac{-3}{2} ; \quad \frac{3}{2} ; \quad \frac{3}{2} ; x^{2}, y^{2}, z^{2}\right],
\end{aligned}
$$

where $F^{(3)}$ is Srivastava's generalized hypergeometric function (see [33]):

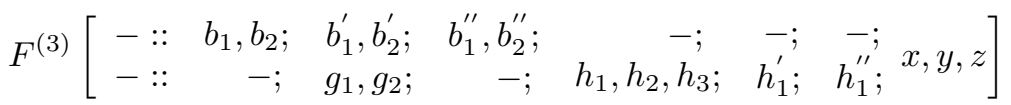

$$
\begin{aligned}
& =\sum_{i, j, k=0}^{\infty} \frac{\left(b_{1}\right)_{i+j}\left(b_{2}\right)_{i+j}\left(b_{1}^{\prime}\right)_{j+k}\left(b_{2}^{\prime}\right)_{j+k}\left(b_{1}^{\prime \prime}\right)_{i+k}\left(b_{2}^{\prime \prime}\right)_{i+k}}{\left(g_{1}\right)_{j+k}\left(g_{2}\right)_{j+k}\left(h_{1}\right)_{i}\left(h_{2}\right)_{i}\left(h_{3}\right)_{i}\left(h_{1}^{\prime}\right)_{j}\left(h_{1}^{\prime \prime}\right)_{k} i ! j ! k !} x^{j} z^{k} \text {. }
\end{aligned}
$$

Conversely, combining the signs of $x, y$ and $z$ in the definition of $H_{A}$, from (2.2) we readily express $F^{(3)}$ in terms of $H_{A}$ 's.

Theorem 2. The following eight relationships between $F^{(3)}$ and $H_{A}$ hold true.

(2.4) $8 F^{(3)}\left[\begin{array}{rrrr}-:: & \frac{a_{2}}{2}, \frac{a_{2}+1}{2} ; & \frac{a_{3}}{2}, \frac{a_{3}+1}{2} ; & \frac{a_{1}}{2}, \frac{a_{1}+1}{2} ; \\ -:: & -; & \frac{c_{2}}{2}, \frac{c_{2}+1}{2} ; & -;\end{array}\right.$

$$
\begin{gathered}
\left.\frac{c_{1}}{2}, \frac{c_{1}+1}{2}, \frac{1}{2} ; \quad-\frac{1}{2} ; \quad \frac{1}{2} ; x^{2}, y^{2}, z^{2}\right] \\
=H_{A}(x, y, z)+H_{A}(-x, y, z)+H_{A}(x, y,-z)+H_{A}(x,-y, z) \\
+H_{A}(-x,-y, z)+H_{A}(-x, y,-z)+H_{A}(x,-y,-z)+H_{A}(-x,-y,-z) ;
\end{gathered}
$$


(2.5)

$$
\begin{aligned}
& \frac{8 a_{1} a_{2} x}{c_{1}} F^{(3)}\left[\begin{array}{lrrrr}
-:: & \frac{a_{2}+1}{2}, \frac{a_{2}+2}{2} ; & \frac{a_{3}}{2}, \frac{a_{3}+1}{2} ; & \frac{a_{1}}{2}, \frac{a_{1}+1}{2} ; \\
-:: & -; & \frac{c_{2}}{2}, \frac{c_{2}+1}{2} ; & -;
\end{array}\right. \\
& \left.\frac{c_{1}+1}{2}, \frac{c_{1}+2}{2}, \frac{3}{2} ; \quad \frac{1}{2} ; \quad \frac{1}{2} ; x^{2}, y^{2}, z^{2}\right] \\
& =H_{A}(x, y, z)-H_{A}(-x, y, z)+H_{A}(x,-y, z)+H_{A}(x, y,-z) \\
& -H_{A}(-x,-y, z)-H_{A}(-x, y,-z)+H_{A}(x,-y,-z)-H_{A}(-x,-y,-z) \text {; }
\end{aligned}
$$

(2.6) $\frac{8 a_{2} a_{3} y}{c_{2}} F^{(3)}\left[\begin{array}{rrrr}-:: & \frac{a_{2}+1}{2}, \frac{a_{2}+2}{2} ; & \frac{a_{3}+1}{2}, \frac{a_{3}+2}{2} ; & \frac{a_{1}}{2}, \frac{a_{1}+1}{2} ; \\ -:: & -; & \frac{c_{2}+1}{2}, \frac{c_{2}+2}{2} ; & -;\end{array}\right.$

$$
\begin{gathered}
\left.\frac{c_{1}}{2}, \frac{c_{1}+1}{2}, \frac{1}{2} ; \quad \frac{3}{2} ; \quad \frac{1}{2} ; x^{2}, y^{2}, z^{2}\right] \\
=H_{A}(x, y, z)+H_{A}(-x, y, z)-H_{A}(x,-y, z)+H_{A}(x, y,-z) \\
-H_{A}(-x,-y, z)+H_{A}(-x, y,-z)-H_{A}(x,-y,-z)-H_{A}(-x,-y,-z) ;
\end{gathered}
$$

(2.7)

$$
\begin{aligned}
& \frac{8 a_{1} a_{3} z}{c_{2}} F^{(3)}\left[\begin{array}{rrrr}
-:: & \frac{a_{2}}{2}, \frac{a_{2}+1}{2} ; & \frac{a_{3}+1}{2}, \frac{a_{3}+2}{2} ; & \frac{a_{1}+1}{2}, \frac{a_{1}+2}{2} ; \\
-:: & -; & \frac{c_{2}+1}{2}, \frac{c_{2}+2}{2} ; & -;
\end{array}\right.
\end{aligned}
$$

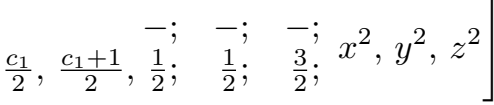

$$
\begin{aligned}
& =H_{A}(x, y, z)+H_{A}(-x, y, z)+H_{A}(x,-y, z)-H_{A}(x, y,-z) \\
& +H_{A}(-x,-y, z)-H_{A}(-x, y,-z)-H_{A}(x,-y,-z)-H_{A}(-x,-y,-z) \text {; }
\end{aligned}
$$

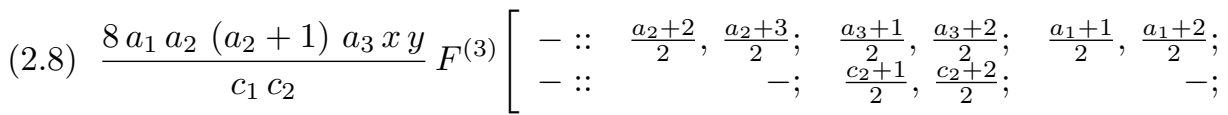

$$
\begin{gathered}
\left.\frac{c_{1}+1}{2}, \frac{c_{1}+2}{2}, \frac{3}{2} ; \frac{3}{2} ; \quad \frac{1}{2} ; x^{2}, y^{2}, z^{2}\right] \\
=H_{A}(x, y, z)-H_{A}(-x, y, z)-H_{A}(x,-y, z)+H_{A}(x, y,-z) \\
+H_{A}(-x,-y, z)-H_{A}(-x, y,-z)-H_{A}(x,-y,-z)+H_{A}(-x,-y,-z) ;
\end{gathered}
$$

$(2.9)$

$$
\begin{aligned}
& \frac{8 a_{1}\left(a_{1}+1\right) a_{2} a_{3} x z}{c_{1} c_{2}} F^{(3)}\left[\begin{array}{rrrr}
-:: & \frac{a_{2}+1}{2}, \frac{a_{2}+2}{2} ; & \frac{a_{3}+1}{2}, \frac{a_{3}+2}{2} ; & \frac{a_{1}+2}{2}, \frac{a_{1}+3}{2} ; \\
-:: & -; & \frac{c_{2}+1}{2}, \frac{c_{2}+2}{2} ; & -;
\end{array}\right. \\
& \left.\frac{c_{1}+1}{2}, \frac{c_{1}+2}{2}, \frac{-3}{2} ; \quad \frac{-1}{2} ; \quad \frac{3}{2} ; x^{2}, y^{2}, z^{2}\right] \\
& =H_{A}(x, y, z)-H_{A}(-x, y, z)+H_{A}(x,-y, z)-H_{A}(x, y,-z) \\
& -H_{A}(-x,-y, z)+H_{A}(-x, y,-z)-H_{A}(x,-y,-z)+H_{A}(-x,-y,-z) \text {; }
\end{aligned}
$$




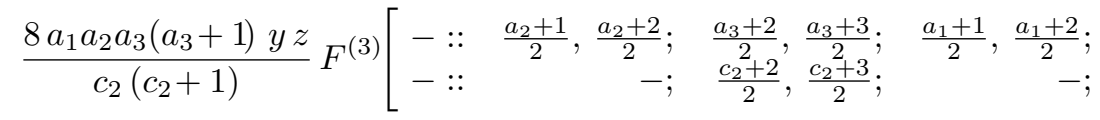

$$
\begin{aligned}
& \left.\frac{c_{1}}{2}, \frac{c_{1}+1}{2}, \frac{-1}{2} ; \quad \frac{-3}{2} ; \quad \frac{3}{2} ; x^{2}, y^{2}, z^{2}\right] \\
& =H_{A}(x, y, z)+H_{A}(-x, y, z)-H_{A}(x,-y, z)-H_{A}(x, y,-z) \\
& -H_{A}(-x,-y, z)-H_{A}(-x, y,-z)+H_{A}(x,-y,-z)+H_{A}(-x,-y,-z) \text {; } \\
& \frac{8 a_{1}\left(a_{1}+1\right) a_{2}\left(a_{2}+1\right) a_{3}\left(a_{3}+1\right) x y z}{c_{1} c_{2}\left(c_{2}+1\right)}
\end{aligned}
$$

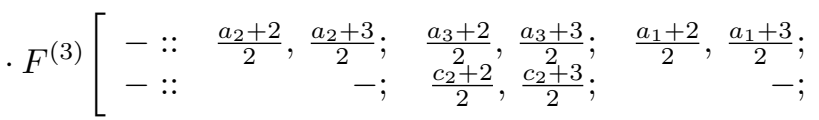

$$
\begin{aligned}
& \left.\frac{c_{1}+1}{2}, \frac{c_{1}+2}{2}, \frac{-3}{2} ; \quad \frac{3}{2} ; \quad \frac{3}{2} ; x^{2}, y^{2}, z^{2}\right] \\
& =H_{A}(x, y, z)-H_{A}(-x, y, z)-H_{A}(x,-y, z)-H_{A}(x, y,-z) \\
& +H_{A}(-x,-y, z)+H_{A}(-x, y,-z)+H_{A}(x,-y,-z)-H_{A}(-x,-y,-z) \text {, }
\end{aligned}
$$

where, for simplicity, $H_{A}(x, y, z):=H_{A}\left(a_{1}, a_{2}, a_{3} ; c_{1}, c_{2} ; x, y, z\right)$.

\section{Limiting Cases}

Here we want to express the triple hypergeometric functions in terms of simpler hypergeometric functions. For this purpose we begin by providing functional relationships between a little simpler function of $H_{A}$ and $F^{(3)}$ as in Corollary 1. Indeed, in order to use the method suggested in [8], employing the following transformations $a_{1} \sim 1 / \varepsilon, x \sim \varepsilon x, z \sim \varepsilon z$ in identities (2.1) and (2.4) to (2.11), and taking the limit of the resulting identities as $\varepsilon \rightarrow 0$, we obtain

Corollary 1. Each of the following relationships holds true.

$$
{ }_{1} H_{A}\left(a_{2}, a_{3} ; c_{1}, c_{2} ; x, y, z\right)
$$

$$
\begin{aligned}
& =F^{(3)}\left[\begin{array}{rrrrrrrr}
-:: & \frac{a_{2}}{2}, \frac{a_{2}+1}{2} ; & \frac{a_{3}}{2}, \frac{a_{3}+1}{2} ; & -; & -; & -; & -; & x^{2} \\
-:: & -; & \frac{c_{2}}{2}, \frac{c_{2}+1}{2} ; & -; & \frac{c_{1}}{2}, \frac{c_{1}+1}{2}, \frac{1}{2} ; & \frac{1}{2} ; & \frac{1}{2} ; & \frac{z^{2}}{4}
\end{array} y^{2}, \frac{1}{4}\right] \\
& +\frac{a_{2} x}{c_{1}} F^{(3)}\left[\begin{array}{rrrr}
-:: & \frac{a_{2}+1}{2}, \frac{a_{2}+2}{2} ; & \frac{a_{3}}{2}, \frac{a_{3}+1}{2} ; & -; \\
-:: & -; & \frac{c_{2}}{2}, \frac{c_{2}+1}{2} ; & -;
\end{array}\right. \\
& \left.\frac{c_{1}+1}{2}, \frac{c_{1}+2}{2}, \frac{3}{2} ; \quad-\frac{1}{2} ; \quad \frac{-1}{2} ; \quad \frac{x^{2}}{4}, y^{2}, \frac{z^{2}}{4}\right]
\end{aligned}
$$




$$
\begin{aligned}
& +\frac{a_{2} a_{3} y}{c_{2}} F^{(3)}\left[\begin{array}{rrrr}
-:: & \frac{a_{2}+1}{2}, \frac{a_{2}+2}{2} ; & \frac{a_{3}+1}{2}, \frac{a_{3}+2}{2} ; & -; \\
-:: & -; & \frac{c_{2}+1}{2}, \frac{c_{2}+2}{2} ; & -;
\end{array}\right. \\
& \left.\frac{c_{1}}{2}, \frac{c_{1}+1}{2}, \frac{1}{2} ; \quad \frac{3}{2} ; \quad \frac{1}{2} ; \quad \frac{x^{2}}{4}, y^{2}, \frac{z^{2}}{4}\right] \\
& +\frac{a_{3} z}{c_{2}} F^{(3)}\left[\begin{array}{rrrr}
-:: & \frac{a_{2}}{2}, \frac{a_{2}+1}{2} ; & \frac{a_{3}+1}{2}, \frac{a_{3}+2}{2} ; & -; \\
-:: & -; & \frac{c_{2}+1}{2}, \frac{c_{2}+2}{2} ; & -;
\end{array}\right. \\
& \left.\frac{c_{1}}{2}, \frac{c_{1}+1}{2}, \frac{-1}{2} ; \quad \frac{1}{2} ; \quad \frac{3}{2} ; \quad \frac{x^{2}}{4}, y^{2}, \frac{z^{2}}{4}\right]
\end{aligned}
$$

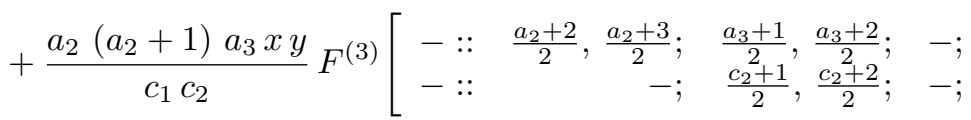

$$
\begin{aligned}
& \left.\frac{c_{1}+1}{2}, \frac{c_{1}+2}{2}, \frac{-3}{2} ; \quad \frac{3}{2} ; \quad \frac{1}{2} ; \quad \frac{x^{2}}{4}, y^{2}, \frac{z^{2}}{4}\right] \\
& +\frac{a_{2} a_{3} x z}{c_{1} c_{2}} F^{(3)}\left[\begin{array}{rrrr}
-:: & \frac{a_{2}+1}{2}, \frac{a_{2}+2}{2} ; & \frac{a_{3}+1}{2}, \frac{a_{3}+2}{2} ; & -; \\
-:: & -; & \frac{c_{2}+1}{2}, \frac{c_{2}+2}{2} ; & -;
\end{array}\right. \\
& \left.\frac{c_{1}+1}{2}, \frac{c_{1}+2}{2}, \frac{-3}{2} ; \quad \frac{1}{2} ; \quad \frac{3}{2} ; \quad \frac{x^{2}}{4}, y^{2}, \frac{z^{2}}{4}\right] \\
& +\frac{a_{2} a_{3}\left(a_{3}+1\right) y z}{c_{2}\left(c_{2}+1\right)} F^{(3)}\left[\begin{array}{rrrr}
-:: & \frac{a_{2}+1}{2}, \frac{a_{2}+2}{2} ; & \frac{a_{3}+2}{2}, \frac{a_{3}+3}{2} ; & -; \\
-:: & -; & \frac{c_{2}+2}{2}, \frac{c_{2}+3}{2} ; & -;
\end{array}\right. \\
& \left.\frac{c_{1}}{2}, \frac{c_{1}+1}{2}, \frac{1}{2} ; \quad \frac{3}{2} ; \quad \frac{3}{2} ; \quad \frac{x^{2}}{4}, y^{2}, \frac{z^{2}}{4}\right] \\
& +\frac{a_{2}\left(a_{2}+1\right) a_{3}\left(a_{3}+1\right) x y z}{c_{1} c_{2}\left(c_{2}+1\right)}
\end{aligned}
$$

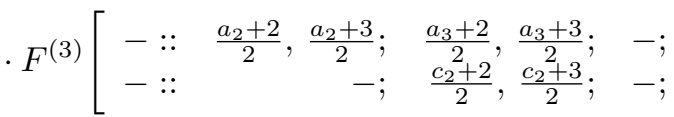

$$
\begin{aligned}
& \left.\frac{c_{1}+1}{2}, \frac{c_{1}+2}{2}, \frac{-3}{2} ; \quad \frac{3}{2} ; \quad \frac{3}{2} ; \quad \frac{x^{2}}{4}, y^{2}, \frac{z^{2}}{4}\right] ;
\end{aligned}
$$

$$
\begin{aligned}
& 8 F^{(3)}\left[\begin{array}{rrrrrrrr}
-:: & \frac{a_{2}}{2}, \frac{a_{2}+1}{2} ; & \frac{a_{3}}{2}, \frac{a_{3}+1}{2} ; & -; & -; & -; & -; & x^{2} \\
-:: & -; & \frac{c_{2}}{2}, \frac{c_{2}+1}{2} ; & -; & \frac{c_{1}}{2}, \frac{c_{1}+1}{2}, \frac{1}{2} ; & \frac{1}{2} ; & \frac{1}{2} ; & \frac{z^{2}}{4}
\end{array} y^{2}, \frac{z^{4}}{4}\right] \\
& ={ }_{1} H_{A}(x, y, z)+{ }_{1} H_{A}(-x, y, z)+{ }_{1} H_{A}(x, y,-z)+{ }_{1} H_{A}(x,-y, z) \\
& +{ }_{1} H_{A}(-x,-y, z)+{ }_{1} H_{A}(-x, y,-z)+{ }_{1} H_{A}(x,-y,-z)+{ }_{1} H_{A}(-x,-y,-z) \text {; }
\end{aligned}
$$


(3.3)

$$
\begin{aligned}
& \frac{8 a_{2} x}{c_{1}} F^{(3)}\left[\begin{array}{rrrr}
-:: & \frac{a_{2}+1}{2}, \frac{a_{2}+2}{2} ; & \frac{a_{3}}{2}, \frac{a_{3}+1}{2} ; & -; \\
-:: & -; & \frac{c_{2}}{2}, \frac{c_{2}+1}{2} ; & -;
\end{array}\right. \\
& \left.\frac{c_{1}+1}{2}, \frac{c_{1}+2}{2}, \frac{3}{2} ; \quad-\frac{1}{2} ; \quad-\frac{1}{2} ; \frac{x^{2}}{4}, y^{2}, \frac{z^{2}}{4}\right] ; \\
& ={ }_{1} H_{A}(x, y, z)-{ }_{1} H_{A}(-x, y, z)+{ }_{1} H_{A}(x,-y, z) \\
& +{ }_{1} H_{A}(x, y,-z)-{ }_{1} H_{A}(-x,-y, z)-{ }_{1} H_{A}(-x, y,-z) \\
& +{ }_{1} H_{A}(x,-y,-z)-{ }_{1} H_{A}(-x,-y,-z) \text {; }
\end{aligned}
$$

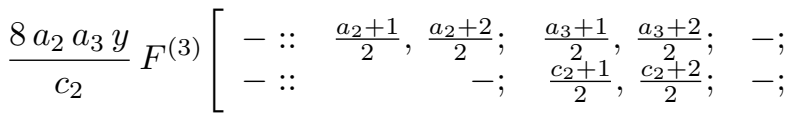

$$
\begin{aligned}
& \left.\frac{c_{1}}{2}, \frac{c_{1}+1}{2}, \frac{1}{2} ; \quad \frac{3}{2} ; \quad \frac{1}{2} ; \frac{x^{2}}{4}, y^{2}, \frac{z^{2}}{4}\right] ; \\
& ={ }_{1} H_{A}(x, y, z)+{ }_{1} H_{A}(-x, y, z)-{ }_{1} H_{A}(x,-y, z) \\
& +{ }_{1} H_{A}(x, y,-z)-{ }_{1} H_{A}(-x,-y, z)+{ }_{1} H_{A}(-x, y,-z) \\
& -{ }_{1} H_{A}(x,-y,-z)-{ }_{1} H_{A}(-x,-y,-z) \text {; }
\end{aligned}
$$

$$
\begin{aligned}
& \frac{8 a_{3} z}{c_{2}} F^{(3)}\left[\begin{array}{rrrr}
-:: & \frac{a_{2}}{2}, \frac{a_{2}+1}{2} ; & \frac{a_{3}+1}{2}, \frac{a_{3}+2}{2} ; & -; \\
-:: & -; & \frac{c_{2}+1}{2}, \frac{c_{2}+2}{2} ; & -;
\end{array}\right. \\
& \left.\frac{c_{1}}{2}, \frac{c_{1}+1}{2}, \frac{1}{2} ; \quad-\frac{1}{2} ; \quad \frac{3}{2} ; \frac{x^{2}}{4}, y^{2}, \frac{z^{2}}{4}\right] ; \\
& ={ }_{1} H_{A}(x, y, z)+{ }_{1} H_{A}(-x, y, z)-{ }_{1} H_{A}(x, y,-z) \\
& +{ }_{1} H_{A}(x,-y, z)+{ }_{1} H_{A}(-x,-y, z)-{ }_{1} H_{A}(-x, y,-z) \\
& -{ }_{1} H_{A}(x,-y,-z)-{ }_{1} H_{A}(-x,-y,-z) \text {; }
\end{aligned}
$$

(3.6)

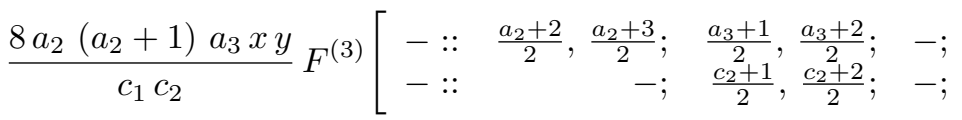

$$
\begin{aligned}
& \left.\frac{c_{1}+1}{2}, \frac{c_{1}+2}{2}, \frac{-3}{2} ; \quad \frac{-3}{2} ; \quad \frac{1}{2} ; \quad \frac{x^{2}}{4}, y^{2}, \frac{z^{2}}{4}\right] \text {; } \\
& ={ }_{1} H_{A}(x, y, z)-{ }_{1} H_{A}(-x, y, z)-{ }_{1} H_{A}(x,-y, z) \\
& +{ }_{1} H_{A}(x, y,-z)+{ }_{1} H_{A}(-x,-y, z)-{ }_{1} H_{A}(-x, y,-z) \\
& -{ }_{1} H_{A}(x,-y,-z)+{ }_{1} H_{A}(-x,-y,-z) \text {; }
\end{aligned}
$$




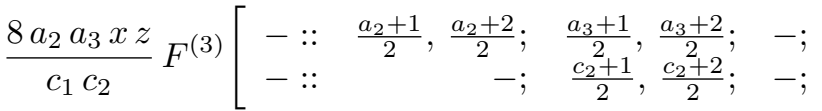

$$
\begin{aligned}
& \left.\frac{c_{1}+1}{2}, \frac{c_{1}+2}{2}, \frac{-3}{2} ; \quad \frac{-}{2} ; \quad \frac{3}{2} ; \quad \frac{x^{2}}{4}, y^{2}, \frac{z^{2}}{4}\right] ; \\
& ={ }_{1} H_{A}(x, y, z)-{ }_{1} H_{A}(-x, y, z)+{ }_{1} H_{A}(x,-y, z) \\
& -{ }_{1} H_{A}(x, y,-z)-{ }_{1} H_{A}(-x,-y, z)+{ }_{1} H_{A}(-x, y,-z) \\
& -{ }_{1} H_{A}(x,-y,-z)+{ }_{1} H_{A}(-x,-y,-z) \text {; }
\end{aligned}
$$

$$
\begin{aligned}
& \frac{8 a_{2} a_{3}\left(a_{3}+1\right) y z}{c_{2}\left(c_{2}+1\right)} F^{(3)}\left[\begin{array}{lrrr}
-:: & \frac{a_{2}+1}{2}, \frac{a_{2}+2}{2} ; & \frac{a_{3}+2}{2}, \frac{a_{3}+3}{2} ; & -; \\
-:: & -; & \frac{c_{2}+2}{2}, \frac{c_{2}+3}{2} ; & -;
\end{array}\right. \\
& \left.\frac{c_{1}}{2}, \frac{c_{1}+1}{2}, \frac{1}{2} ; \quad-\frac{3}{2} ; \quad \frac{3}{2} ; \quad \frac{x^{2}}{4}, y^{2}, \frac{z^{2}}{4}\right] ; \\
& ={ }_{1} H_{A}(x, y, z)+{ }_{1} H_{A}(-x, y, z)-{ }_{1} H_{A}(x,-y, z) \\
& -{ }_{1} H_{A}(x, y,-z)-{ }_{1} H_{A}(-x,-y, z)-{ }_{1} H_{A}(-x, y,-z) \\
& +{ }_{1} H_{A}(x,-y,-z)+{ }_{1} H_{A}(-x,-y,-z) \text {; }
\end{aligned}
$$

$$
\begin{aligned}
& \frac{8 a_{2}\left(a_{2}+1\right) a_{3}\left(a_{3}+1\right) x y z}{c_{1} c_{2}\left(c_{2}+1\right)} F^{(3)}\left[\begin{array}{rrrr}
-:: & \frac{a_{2}+2}{2}, \frac{a_{2}+3}{2} ; & \frac{a_{3}+2}{2}, \frac{a_{3}+3}{2} ; & -; \\
-:: & -; & \frac{c_{2}+2}{2}, \frac{c_{2}+3}{2} ; & -;
\end{array}\right. \\
& \left.\frac{c_{1}+1}{2}, \frac{c_{1}+2}{2}, \frac{-3}{2} ; \quad-\frac{3}{2} ; \quad \frac{3}{2} ; \frac{x^{2}}{4}, y^{2}, \frac{z^{2}}{4}\right] \\
& ={ }_{1} H_{A}(x, y, z)-{ }_{1} H_{A}(-x, y, z)-{ }_{1} H_{A}(x,-y, z) \\
& -{ }_{1} H_{A}(x, y,-z)+{ }_{1} H_{A}(-x,-y, z)+{ }_{1} H_{A}(-x, y,-z) \\
& +{ }_{1} H_{A}(x,-y,-z)-{ }_{1} H_{A}(-x,-y,-z) \text {, }
\end{aligned}
$$

where ${ }_{1} H_{A}(x, y, z):={ }_{1} H_{A}\left(a_{2}, a_{3} ; c_{1}, c_{2} ; x, y, z\right)$ and

$$
\begin{aligned}
{ }_{1} H_{A} & \left(a_{2}, a_{3} ; c_{1}, c_{2} ; x, y, z\right)=\lim _{\varepsilon \rightarrow 0} H_{A}\left(\frac{1}{\varepsilon}, a_{2}, a_{3} ; c_{1}, c_{2} ; \varepsilon x, y, \varepsilon z\right) \\
= & \sum_{m, n, p=0}^{\infty} \frac{\left(a_{2}\right)_{m+n}\left(a_{3}\right)_{n+p}}{\left(c_{1}\right)_{m}\left(c_{2}\right)_{n+p} m ! n ! p !} x^{m} y^{n} z^{p} .
\end{aligned}
$$

For further specializations we start with observing the following limits: 
Lemma 1. Each of the following relationships holds true.

$$
\begin{aligned}
& \lim _{\varepsilon \rightarrow 0} F^{(3)}\left[\begin{array}{rrrrr}
-:: & \frac{a_{2}}{2}, \frac{a_{2}+1}{2} ; & \frac{a_{3}}{2}, \frac{a_{3}+1}{2} ; & \frac{1}{2 \varepsilon}, \frac{1+\varepsilon}{2 \varepsilon} ; \\
-:: & -; & \frac{c_{2}}{2}, \frac{c_{2}+1}{2} ; & -;
\end{array}\right. \\
& \left.\frac{c_{1}}{2}, \frac{c_{1}+1}{2}, \frac{1}{2} ; \quad-\frac{1}{2} ; \quad-\frac{1}{2} ; \quad(\varepsilon x)^{2}, y^{2},(\varepsilon z)^{2}\right] \\
& =F^{(3)}\left[\begin{array}{rrrr}
-:: & \frac{a_{2}}{2}, \frac{a_{2}+1}{2} ; & \frac{a_{3}}{2}, \frac{a_{3}+1}{2} ; & -; \\
-:: & -; & \frac{c_{2}}{2}, \frac{c_{2}+1}{2} ; & -;
\end{array}\right. \\
& \left.\frac{c_{1}}{2}, \frac{c_{1}+1}{2}, \frac{1}{2} ; \quad \frac{1}{2} ; \quad \frac{1}{2} ; \quad \frac{x^{2}}{4}, y^{2}, \frac{z^{2}}{4}\right] ;
\end{aligned}
$$

$$
\lim _{\varepsilon \rightarrow 0}{ }_{1} H_{A}\left(\frac{1}{\varepsilon}, a_{3} ; c_{1}, c_{2} ; \varepsilon x, \varepsilon y, z\right)={ }_{0} F_{1}\left(c_{1} ; x\right){ }_{1} F_{1}\left(a_{3} ; c_{2} ; y+z\right)
$$

$$
\begin{aligned}
& \lim _{\varepsilon \rightarrow 0} F^{(3)}\left[\begin{array}{rrrr}
-:: & \frac{1}{2 \varepsilon}, \frac{1+\varepsilon}{2 \varepsilon} ; & \frac{a_{3}}{2}, \frac{a_{3}+1}{2} ; & -; \\
-:: & -; & \frac{c_{2}}{2}, \frac{c_{2}+1}{2} ; & -;
\end{array}\right. \\
& \left.\frac{c_{1}}{2}, \frac{c_{1}+1}{2}, \frac{1}{2} ; \quad-\frac{1}{2} ; \quad \frac{1}{2} ; \quad \frac{(\varepsilon x)^{2}}{4},(\varepsilon y)^{2}, \frac{z^{2}}{4}\right] \\
& ={ }_{0} F_{3}\left(\frac{c_{1}}{2}, \frac{c_{1}+1}{2}, \frac{1}{2} ; \frac{x^{2}}{16}\right) F_{2: 1 ; 1}^{2: 0 ; 0}\left[\frac{\frac{a_{3}}{2}, \frac{a_{3}+1}{\frac{c_{2}}{2}}, \frac{c_{2}+1}{2}:}{\frac{c_{2}}{2}} \quad \frac{-}{2} ; \frac{y^{2}}{4}, \frac{z^{2}}{4}\right] \text {, }
\end{aligned}
$$

where $F_{2: 1 ; 1}^{2: 0 ; 0}$ is a hypergeometric Kampé de Fériet function $($ see $[3,33])$ of two variables defined by

$$
F_{2: 1 ; 1}^{2: 0 ; 0}\left[\begin{array}{ccc}
a_{1}, a_{2}: & -; & -; \\
c_{1}, c_{2}: & d ; & e ;
\end{array} y, z\right]=\sum_{n, p=0}^{\infty} \frac{\left(a_{1}\right)_{n+p}\left(a_{2}\right)_{n+p}}{\left(c_{1}\right)_{n+p}\left(c_{2}\right)_{n+p}(d)_{n}(e)_{p} n ! p !} y^{n} z^{p}
$$

and ${ }_{p} F_{q}$ denotes the generalized hypergeometric function (see [33]).

Setting $a_{2} \sim 1 / \varepsilon, x \sim \varepsilon x, y \sim \varepsilon y$, in (3.1) to (3.9), and taking the limit of the resulting identities as $\varepsilon \rightarrow 0$, and using the identities in Lemma1, we get 
Corollary 2. Each of the following relationships holds true.

$$
\begin{aligned}
& { }_{0} F_{1}\left(c_{1} ; x\right){ }_{1} F_{1}\left(a_{3} ; c_{2} ; y+z\right) \\
& =\left[{ }_{0} F_{3}\left(\frac{c_{1}}{2}, \frac{c_{1}+1}{2}, \frac{1}{2} ; \frac{x^{2}}{16}\right)+\frac{x}{c_{1}}{ }_{0} F_{3}\left(\frac{c_{1}+1}{2}, \frac{c_{1}+2}{2}, \frac{3}{2} ; \frac{x^{2}}{16}\right)\right]
\end{aligned}
$$

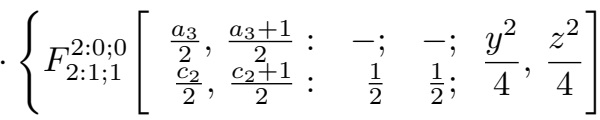

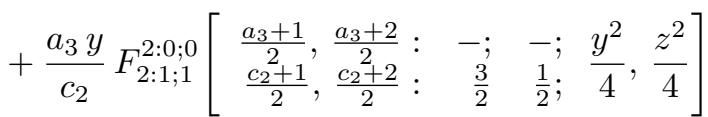

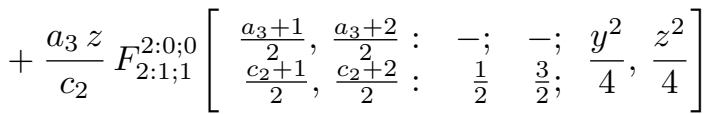

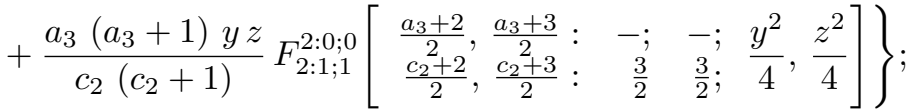

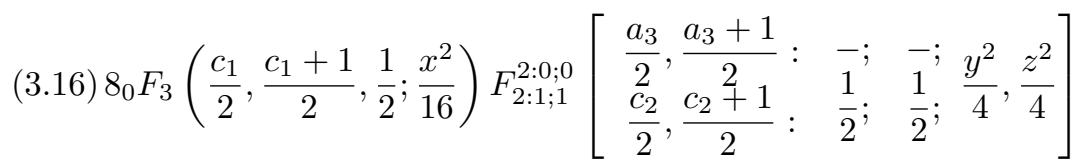

$$
=\left[{ }_{0} F_{1}\left(c_{1} ; x\right)+{ }_{0} F_{1}\left(c_{1} ;-x\right)\right]
$$

$\cdot\left[{ }_{1} F_{1}\left(a_{3} ; c_{2} ; y+z\right)+{ }_{1} F_{1}\left(a_{3} ; c_{2} ; y-z\right)+{ }_{1} F_{1}\left(a_{3} ; c_{2} ;-y+z\right)+{ }_{1} F_{1}\left(a_{3} ; c_{2} ;-y-z\right)\right]$;

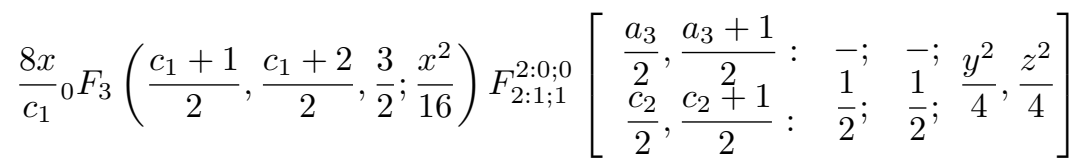

$$
=\left[{ }_{0} F_{1}\left(c_{1} ; x\right)-{ }_{0} F_{1}\left(c_{1} ;-x\right)\right]
$$

$\cdot\left[{ }_{1} F_{1}\left(a_{3} ; c_{2} ; y+z\right)+{ }_{1} F_{1}\left(a_{3} ; c_{2} ; y-z\right)+{ }_{1} F_{1}\left(a_{3} ; c_{2} ;-y+z\right)+{ }_{1} F_{1}\left(a_{3} ; c_{2} ;-y-z\right)\right]$;

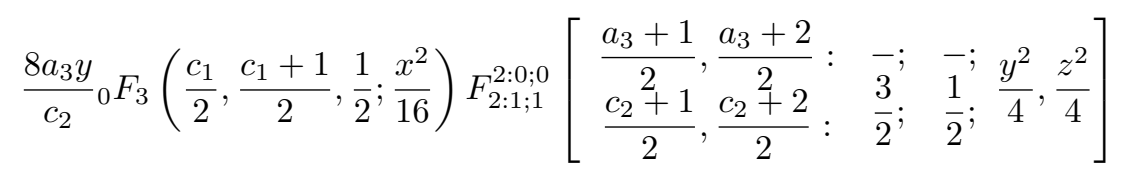$$
=\left[{ }_{0} F_{1}\left(c_{1} ; x\right)+{ }_{0} F_{1}\left(c_{1} ;-x\right)\right]
$$$$
\cdot\left[{ }_{1} F_{1}\left(a_{3} ; c_{2} ; y+z\right)-{ }_{1} F_{1}\left(a_{3} ; c_{2} ;-y+z\right)+{ }_{1} F_{1}\left(a_{3} ; c_{2} ; y-z\right)-{ }_{1} F_{1}\left(a_{3} ; c_{2} ;-y-z\right)\right] \text {; }
$$

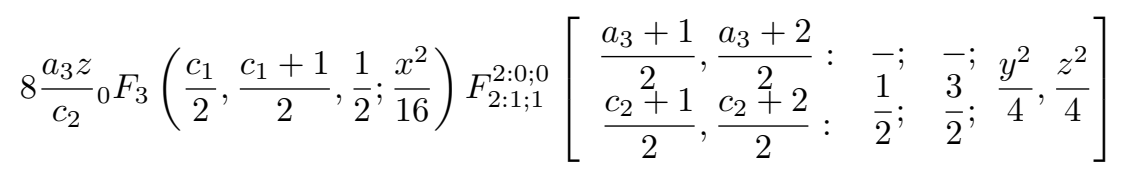


$=\left[{ }_{0} F_{1}\left(c_{1} ; x\right)+{ }_{0} F_{1}\left(c_{1} ;-x\right)\right]$

$\cdot\left[{ }_{1} F_{1}\left(a_{3} ; c_{2} ; y+z\right)+{ }_{1} F_{1}\left(a_{3} ; c_{2} ;-y+z\right)-{ }_{1} F_{1}\left(a_{3} ; c_{2} ; y-z\right)-{ }_{1} F_{1}\left(a_{3} ; c_{2} ;-y-z\right)\right] ;$

$$
\begin{aligned}
& \frac{8 a_{3} x y}{c_{1} c_{2}}{ }_{0} F_{3}\left(\frac{c_{1}+1}{2}, \frac{c_{1}+2}{2}, \frac{3}{2} ; \frac{x^{2}}{16}\right)
\end{aligned}
$$

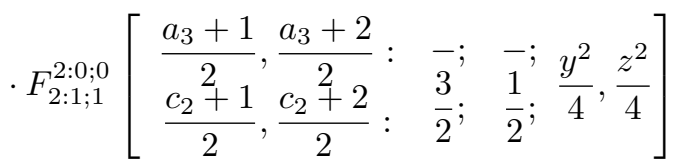

$=\left[{ }_{0} F_{1}\left(c_{1} ; x\right)-{ }_{0} F_{1}\left(c_{1} ;-x\right)\right]$

$\cdot\left[{ }_{1} F_{1}\left(a_{3} ; c_{2} ; y+z\right)-{ }_{1} F_{1}\left(a_{3} ; c_{2} ;-y+z\right)+{ }_{1} F_{1}\left(a_{3} ; c_{2} ; y-z\right)-{ }_{1} F_{1}\left(a_{3} ; c_{2} ;-y-z\right)\right]$;

$$
\begin{aligned}
& \frac{8 a_{3} x z}{c_{1} c_{2}}{ }_{0} F_{3}\left(\frac{c_{1}+1}{2}, \frac{c_{1}+2}{2}, \frac{3}{2} ; \frac{x^{2}}{16}\right) \\
& \cdot F_{2: 1 ; 1}^{2: 0 ; 0}\left[\begin{array}{ll}
\frac{a_{3}+1}{2}, \frac{a_{3}+2}{2}: & -\frac{1}{2} ; \frac{y^{2}}{4}, \frac{z^{2}}{4} \\
\frac{c_{2}+1}{2}: \frac{c_{2}+2}{2} ; & \frac{1}{2} ; \frac{1}{4}
\end{array}\right.
\end{aligned}
$$

$=\left[{ }_{0} F_{1}\left(c_{1} ; x\right)-{ }_{0} F_{1}\left(c_{1} ;-x\right)\right]$

$\cdot\left[{ }_{1} F_{1}\left(a_{3} ; c_{2} ; y+z\right)+{ }_{1} F_{1}\left(a_{3} ; c_{2} ;-y+z\right)-{ }_{1} F_{1}\left(a_{3} ; c_{2} ; y-z\right)-{ }_{1} F_{1}\left(a_{3} ; c_{2} ;-y-z\right)\right] ;$

$$
\begin{aligned}
& \frac{8 a_{3}\left(a_{3}+1\right) y z}{c_{2}\left(c_{2}+1\right)}{ }_{0} F_{3}\left(\frac{c_{1}}{2}, \frac{c_{1}+1}{2}, \frac{1}{2} ; \frac{x^{2}}{16}\right) \\
& \cdot F_{2: 1 ; 1}^{2: 0 ; 0}\left[\frac{\frac{a_{3}+2}{2}, \frac{a_{3}+3}{2}:}{\frac{c_{2}+2}{2}, \frac{c_{2}+3}{2}:} ; \frac{3}{2} ; \frac{3}{2} ; \frac{y^{2}}{4}, \frac{z^{2}}{4}\right]
\end{aligned}
$$

$=\left[{ }_{0} F_{1}\left(c_{1} ; x\right)+{ }_{0} F_{1}\left(c_{1} ;-x\right)\right]$

$\cdot\left[{ }_{1} F_{1}\left(a_{3} ; c_{2} ; y+z\right)-{ }_{1} F_{1}\left(a_{3} ; c_{2} ;-y+z\right)-{ }_{1} F_{1}\left(a_{3} ; c_{2} ; y-z\right)+{ }_{1} F_{1}\left(a_{3} ; c_{2} ;-y-z\right)\right]$;

$$
\begin{aligned}
& (3.23) \frac{8 a_{3}\left(a_{3}+1\right) x y z}{c_{1} c_{2}\left(c_{2}+1\right)}{ }_{0} F_{3}\left(\frac{c_{1}+1}{2}, \frac{c_{1}+2}{2}, \frac{3}{2} ; \frac{x^{2}}{16}\right) \\
& \cdot F_{2: 1 ; 1}^{2: 0 ; 0}\left[\frac{\left.\frac{a_{3}+2}{2}, \frac{a_{3}+3}{2}: \frac{-}{2} ; \frac{-}{3} ; \frac{y^{2}}{2}, \frac{z^{2}}{4}\right)}{2} ; \frac{3}{2} ; \frac{c_{2}+3}{4}\right] \\
& =\left[{ }_{0} F_{1}\left(c_{1} ; x\right)-{ }_{0} F_{1}\left(c_{1} ;-x\right)\right] \\
& \cdot\left[{ }_{1} F_{1}\left(a_{3} ; c_{2} ; y+z\right)-{ }_{1} F_{1}\left(a_{3} ; c_{2} ;-y+z\right)-{ }_{1} F_{1}\left(a_{3} ; c_{2} ; y-z\right)+{ }_{1} F_{1}\left(a_{3} ; c_{2} ;-y-z\right)\right] .
\end{aligned}
$$$$
=\left[{ }_{0} F_{1}\left(c_{1} ; x\right)-{ }_{0} F_{1}\left(c_{1} ;-x\right)\right]
$$

Setting $a_{3} \sim 1 / \varepsilon, y \sim \varepsilon y, z \sim \varepsilon z$ in (3.15) to (3.23) and taking the limit of the resulting identities as $\varepsilon \rightarrow 0$, we find 
Corollary 3. Each of the following relationships holds true.

$$
\begin{aligned}
& { }_{0} F_{1}\left(c_{1} ; x\right){ }_{0} F_{1}\left(c_{2} ; y+z\right) \\
& =\left[{ }_{0} F_{3}\left(\frac{c_{1}}{2}, \frac{c_{1}+1}{2}, \frac{1}{2} ; \frac{x^{2}}{16}\right)+\frac{1}{c_{1}} x F_{3}\left(\frac{c_{1}+1}{2}, \frac{c_{1}+2}{2}, \frac{3}{2} ; \frac{x^{2}}{16}\right)\right]
\end{aligned}
$$

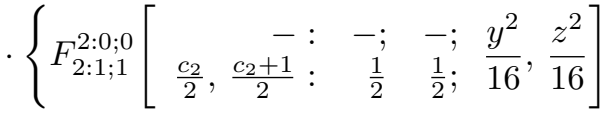

$$
\begin{aligned}
& +\frac{y}{c_{2}} F_{2: 1 ; 1}^{2: 0 ; 0}\left[\begin{array}{rrrr}
-: & -; & -; & y^{2} \\
\frac{c_{2}+1}{2}, \frac{c_{2}+2}{2}: & \frac{3}{2} & \frac{1}{2} ; & \frac{z^{2}}{16} \\
16
\end{array}\right] \\
& +\frac{z}{c_{2}} F_{2: 1 ; 1}^{2: 0 ; 0}\left[\begin{array}{rrrr}
-: & -; & -; & y^{2} \\
\frac{c_{2}+1}{2}, \frac{c_{2}+2}{2}: & \frac{1}{2} & \frac{3}{2} ; & \frac{z^{2}}{16}
\end{array}\right] \\
& \left.+\frac{y z}{c_{2}\left(c_{2}+1\right)} F_{2: 1 ; 1}^{2: 0 ; 0}\left[\begin{array}{rrrr}
-: & -; & -; & y^{2} \\
\frac{c_{2}+2}{2}, \frac{c_{2}+3}{2}: & \frac{3}{2} & \frac{3}{2} ; & \frac{z^{2}}{16}, \\
16
\end{array}\right]\right\}
\end{aligned}
$$

$$
\begin{aligned}
& \frac{8 x}{c_{1}}{ }_{0} F_{3}\left(\frac{c_{1}+1}{2}, \frac{c_{1}+2}{2}, \frac{3}{2} ; \frac{x^{2}}{16}\right) F_{2: 1 ; 1}^{0: 0 ; 0}\left[\frac{c_{2}}{2}, \frac{c_{2}+\frac{-}{2}:}{2}: \frac{-}{2} ; \frac{1}{2} ; \frac{y^{2}}{16}, \frac{z^{2}}{16}\right] \\
= & {\left[{ }_{0} F_{1}\left(c_{1} ; x\right)-{ }_{0} F_{1}\left(c_{1} ;-x\right)\right] } \\
\cdot & {\left[{ }_{0} F_{1}\left(c_{2} ; y+z\right)+{ }_{0} F_{1}\left(c_{2} ; y-z\right)+{ }_{0} F_{1}\left(c_{2} ;-y+z\right)+{ }_{0} F_{1}\left(c_{2} ;-y-z\right)\right] ; }
\end{aligned}
$$

$$
\begin{aligned}
& \frac{8 y}{c_{2}}{ }_{0} F_{3}\left(\frac{c_{1}}{2}, \frac{c_{1}+1}{2}, \frac{1}{2} ; \frac{x^{2}}{16}\right) F_{2: 1 ; 1}^{0: 0 ; 0}\left[\frac{c_{2}+1}{2}, \frac{c_{2}+2}{2}: \frac{3}{2} ; \frac{1}{2} ; \frac{y^{2}}{16}, \frac{z^{2}}{16}\right] \\
= & {\left[{ }_{0} F_{1}\left(c_{1} ; x\right)+{ }_{0} F_{1}\left(c_{1} ;-x\right)\right] } \\
& \cdot\left[{ }_{0} F_{1}\left(c_{2} ; y+z\right)-{ }_{0} F_{1}\left(c_{2} ;-y+z\right)+{ }_{0} F_{1}\left(c_{2} ; y-z\right)-{ }_{0} F_{1}\left(c_{2} ;-y-z\right)\right] ; \\
= & \frac{8 z}{c_{2}}{ }_{0} F_{3}\left(\frac{c_{1}}{2}, \frac{c_{1}+1}{2}, \frac{1}{2} ; \frac{x^{2}}{16}\right) F_{2: 1 ; 1}^{0: 0 ; 0}\left[\frac{c_{2}+1}{2}, \frac{c_{2}+2}{2}: \frac{-1}{2} ; \frac{3}{2} ; \frac{y^{2}}{16}, \frac{z^{2}}{16}\right] \\
= & {\left[{ }_{0} F_{1}\left(c_{1} ; x\right)+{ }_{0} F_{1}\left(c_{1} ;-x\right)\right] } \\
& \cdot\left[{ }_{0} F_{1}\left(c_{2} ; y+z\right)+{ }_{0} F_{1}\left(c_{2} ;-y+z\right)-{ }_{0} F_{1}\left(c_{2} ; y-z\right)-{ }_{0} F_{1}\left(c_{2} ;-y-z\right)\right] ;
\end{aligned}
$$

$$
\begin{aligned}
& \frac{8 x y}{c_{1} c_{2}}{ }_{0} F_{3}\left(\frac{c_{1}+1}{2}, \frac{c_{1}+2}{2}, \frac{3}{2} ; \frac{x^{2}}{16}\right) \\
& \cdot F_{2: 1 ; 1}^{0: 0 ; 0}\left[\frac{c_{2}+1}{2}, \frac{c_{2}+2}{2}: \frac{-}{2} ; \frac{1}{2} ; \frac{y^{2}}{16}, \frac{z^{2}}{16}\right]
\end{aligned}
$$




$$
\begin{aligned}
= & {\left[{ }_{0} F_{1}\left(c_{1} ; x\right)-{ }_{0} F_{1}\left(c_{1} ;-x\right)\right] } \\
\cdot & {\left[{ }_{0} F_{1}\left(c_{2} ; y+z\right)-{ }_{0} F_{1}\left(c_{2} ;-y+z\right)+{ }_{0} F_{1}\left(c_{2} ; y-z\right)-{ }_{0} F_{1}\left(c_{2} ;-y-z\right)\right] ; }
\end{aligned}
$$

$$
\begin{aligned}
& \frac{8 x z}{c_{1} c_{2}}{ }_{0} F_{3}\left(\frac{c_{1}+1}{2}, \frac{c_{1}+2}{2}, \frac{3}{2} ; \frac{x^{2}}{16}\right) \\
& \cdot F_{2: 1 ; 1}^{0: 0 ; 0}\left[\frac{c_{2}+1}{2}, \frac{c_{2}+\frac{-}{2}}{2}: \frac{1}{2} ; \frac{3}{2} ; \frac{y^{2}}{16}, \frac{z^{2}}{16}\right]
\end{aligned}
$$

$$
\begin{aligned}
= & {\left[{ }_{0} F_{1}\left(c_{1} ; x\right)-{ }_{0} F_{1}\left(c_{1} ;-x\right)\right] } \\
\cdot & {\left[{ }_{0} F_{1}\left(c_{2} ; y+z\right)+{ }_{0} F_{1}\left(c_{2} ;-y+z\right)-{ }_{0} F_{1}\left(c_{2} ; y-z\right)-{ }_{0} F_{1}\left(c_{2} ;-y-z\right)\right] ; }
\end{aligned}
$$

$$
\begin{aligned}
& \frac{8 y z}{c_{2}\left(c_{2}+1\right)}{ }_{0} F_{3}\left(\frac{c_{1}}{2}, \frac{c_{1}+1}{2}, \frac{1}{2} ; \frac{x^{2}}{16}\right) \\
& \cdot F_{2: 1 ; 1}^{0: 0 ; 0}\left[\frac{c_{2}+2}{2}, \frac{c_{2}+3}{2}: \frac{-3}{2} ; \quad \frac{3}{2} ; \frac{y^{2}}{16}, \frac{z^{2}}{16}\right] \\
& =\left[{ }_{0} F_{1}\left(c_{1} ; x\right)+{ }_{0} F_{1}\left(c_{1} ;-x\right)\right] \\
& \text { - }\left[{ }_{0} F_{1}\left(c_{2} ; y+z\right)-{ }_{0} F_{1}\left(c_{2} ;-y+z\right)-{ }_{0} F_{1}\left(c_{2} ; y-z\right)+{ }_{0} F_{1}\left(c_{2} ;-y-z\right)\right] ; \\
& \frac{8 x y z}{c_{1} c_{2}\left(c_{2}+1\right)}{ }_{0} F_{3}\left(\frac{c_{1}+1}{2}, \frac{c_{1}+2}{2}, \frac{3}{2} ; \frac{x^{2}}{16}\right) \\
& \cdot F_{2: 1 ; 1}^{0: 0 ; 0}\left[\frac{c_{2}+2}{2}, \frac{c_{2}+3}{2}: \frac{-3}{2} ; \quad \frac{3}{2} ; \frac{y^{2}}{16}, \frac{z^{2}}{16}\right] \\
& =\left[{ }_{0} F_{1}\left(c_{1} ; x\right)-{ }_{0} F_{1}\left(c_{1} ;-x\right)\right] \\
& \cdot\left[{ }_{0} F_{1}\left(c_{2} ; y+z\right)-{ }_{0} F_{1}\left(c_{2} ;-y+z\right)-{ }_{0} F_{1}\left(c_{2} ; y-z\right)+{ }_{0} F_{1}\left(c_{2} ;-y-z\right)\right] \text {. }
\end{aligned}
$$

\section{Special cases}

For certain special cases of some identities in the previous sections, we introduce the case $a_{3}=c_{2}$ of $H_{A}$ as in the following lemma.

Lemma 2. The function $H_{A}$ when $a_{3}=c_{2}$ is seen to reduce to a Gauss hypergeometric function ${ }_{2} F_{1}=F$ :

$$
\begin{aligned}
& H_{A}\left(a_{1}, a_{2}, a_{3} ; c_{1}, a_{3} ; x, y, z\right) \\
= & (1-y)^{-a_{2}}(1-z)^{-a_{1}} F\left(a_{1}, a_{2} ; c_{1} ; \frac{x}{(1-y)(1-z)}\right) .
\end{aligned}
$$

Setting $a_{3}=c_{2}$ in (2.2) and (2.4) to (2.11) and considering (4.1), we obtain 
Corollary 4. Each of the following relationships holds true.

$$
\begin{aligned}
& (1-y)^{-a_{2}}(1-z)^{-a_{1}} F\left(a_{1}, a_{2} ; c_{1} ; \frac{x}{(1-y)(1-z)}\right) \\
& =F^{(3)}\left[\begin{array}{rrrrrrrr}
-:: & \frac{a_{2}}{2}, \frac{a_{2}+1}{2} ; & -; & \frac{a_{1}}{2}, \frac{a_{1}+1}{2} ; & -; & -; & -; & x^{2}, y^{2}, z^{2} \\
-: & -; & -; & -; & \frac{c_{1}}{2}, \frac{c_{1}+1}{2}, \frac{1}{2} ; & \frac{1}{2} ; & \frac{1}{2} ;
\end{array}\right.
\end{aligned}
$$

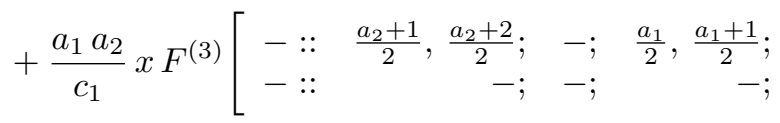

$$
\begin{aligned}
& \left.\frac{c_{1}+1}{2}, \frac{c_{1}+2}{2}, \frac{3}{2} ; \quad-\frac{1}{2} ; \quad-\frac{1}{2} ; x^{2}, y^{2}, z^{2}\right] \\
& +a_{2} y F^{(3)}\left[\begin{array}{rrrrr}
-:: & \frac{a_{2}+1}{2}, \frac{a_{2}+2}{2} ; & -; & \frac{a_{1}}{2}, \frac{a_{1}+1}{2} ; \\
-:: & -; & -; & -;
\end{array}\right. \\
& \left.\frac{c_{1}}{2}, \frac{c_{1}+1}{2}, \frac{1}{2} ; \quad-\frac{3}{2} ; \quad-\frac{1}{2} ; x^{2}, y^{2}, z^{2}\right] \\
& +a_{1} z F^{(3)}\left[\begin{array}{rrrr}
-:: & \frac{a_{2}}{2}, \frac{a_{2}+1}{2} ; & -; & \frac{a_{1}+1}{2}, \frac{a_{1}+2}{2} ; \\
-:: & -; & -; & -;
\end{array}\right. \\
& \left.\frac{c_{1}}{2}, \frac{c_{1}+1}{2}, \frac{1}{2} ; \quad \frac{1}{2} ; \quad \frac{3}{2} ; x^{2}, y^{2}, z^{2}\right]
\end{aligned}
$$

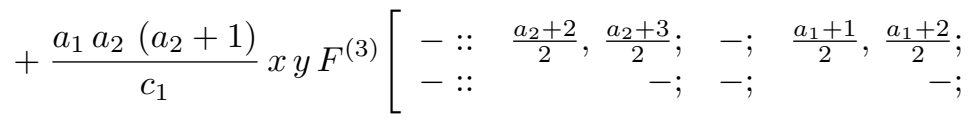

$$
\begin{aligned}
& \left.\frac{c_{1}+1}{2}, \frac{c_{1}+2}{2}, \frac{3}{2} ; \quad \frac{3}{2} ; \quad \frac{1}{2} ; x^{2}, y^{2}, z^{2}\right] \\
& +\frac{a_{1}\left(a_{1}+1\right) a_{2}}{c_{1}} x z F^{(3)}\left[\begin{array}{rrrr}
-:: & \frac{a_{2}+1}{2}, \frac{a_{2}+2}{2} ; & -; & \frac{a_{1}+2}{2}, \frac{a_{1}+3}{2} ; \\
-:: & -; & -; & -;
\end{array}\right. \\
& \left.\frac{c_{1}+1}{2}, \frac{c_{1}+2}{2}, \frac{3}{2} ; \quad \frac{1}{2} ; \quad \frac{3}{2} ; x^{2}, y^{2}, z^{2}\right] \\
& +a_{1} a_{2} y z F^{(3)}\left[\begin{array}{rrrr}
-:: & \frac{a_{2}+1}{2}, \frac{a_{2}+2}{2} ; & -; & \frac{a_{1}+1}{2}, \frac{a_{1}+2}{2} \text {; } \\
-:: & -; & -; & -;
\end{array}\right. \\
& \left.\frac{c_{1}}{2}, \frac{c_{1}+1}{2}, \frac{1}{2} ; \quad \frac{3}{2} ; \quad \frac{3}{2} ; x^{2}, y^{2}, z^{2}\right]
\end{aligned}
$$




$$
\begin{aligned}
& +\frac{a_{1}\left(a_{1}+1\right) a_{2}\left(a_{2}+1\right)}{c_{1}} x y z F^{(3)}\left[\begin{array}{lrrrr}
-:: & \frac{a_{2}+2}{2}, \frac{a_{2}+3}{2} ; & -; & \frac{a_{1}+2}{2}, & \frac{a_{1}+3}{2} ; \\
-:: & -; & -; &
\end{array}\right. \\
& \left.\frac{c_{1}+1}{2}, \frac{c_{1}+2}{2}, \frac{-3}{2} ; \quad \frac{-3}{2} ; \quad \frac{3}{2} ; x^{2}, y^{2}, z^{2}\right] \text {; }
\end{aligned}
$$

(4.3) $8 F^{(3)}\left[\begin{array}{rrrrrrr}-:: & \frac{a_{2}}{2}, \frac{a_{2}+1}{2} ; & -; & \frac{a_{1}}{2}, \frac{a_{1}+1}{2} ; & -; & -; & -; \\ -:: & -; & -; & -; & \frac{c_{1}}{2}, \frac{c_{1}+1}{2}, \frac{1}{2} ; & \frac{1}{2} ; & \frac{1}{2} ;\end{array} x^{2}, y^{2}, z^{2}\right]$

$$
\begin{aligned}
= & (1-y)^{-a_{2}}(1-z)^{-a_{1}} \\
& \cdot\left[F\left(a_{1}, a_{2} ; c_{1} ; \frac{x}{(1-y)(1-z)}\right)+F\left(a_{1}, a_{2} ; c_{1} ; \frac{-x}{(1-y)(1-z)}\right)\right] \\
+ & (1-y)^{-a_{2}}(1+z)^{-a_{1}} \\
& \cdot\left[F\left(a_{1}, a_{2} ; c_{1} ; \frac{x}{(1-y)(1+z)}\right)+F\left(a_{1}, a_{2} ; c_{1} ; \frac{-x}{(1+y)(1-z)}\right)\right] \\
+ & (1+y)^{-a_{2}}(1-z)^{-a_{1}} \\
& \cdot\left[F\left(a_{1}, a_{2} ; c_{1} ; \frac{x}{(1+y)(1-z)}\right)+F\left(a_{1}, a_{2} ; c_{1} ; \frac{-x}{(1-y)(1+z)}\right)\right] \\
+ & (1+y)^{-a_{2}}(1+z)^{-a_{1}} \\
& \cdot\left[F\left(a_{1}, a_{2} ; c_{1} ; \frac{x}{(1+y)(1+z)}\right)+F\left(a_{1}, a_{2} ; c_{1} ; \frac{-x}{(1+y)(1+z)}\right)\right] ;
\end{aligned}
$$

$$
\begin{array}{r}
\frac{8 a_{1} a_{2}}{c_{1}} x F^{(3)}\left[\begin{array}{rrrrr}
-:: & \frac{a_{2}+1}{2}, \frac{a_{2}+2}{2} ; & -; & \frac{a_{1}}{2}, \frac{a_{1}+1}{2} ; \\
-:: & -; & -; & -; \\
& \frac{c_{1}+1}{2}, \frac{c_{1}+2}{2}, \frac{3}{2} ; & -\frac{1}{2} ; & -\frac{1}{2} ; x^{2}, y^{2}, z^{2}
\end{array}\right]
\end{array}
$$

$$
\begin{aligned}
= & (1-y)^{-a_{2}}(1-z)^{-a_{1}} \\
& \cdot\left[F\left(a_{1}, a_{2} ; c_{1} ; \frac{x}{(1-y)(1-z)}\right)+F\left(a_{1}, a_{2} ; c_{1} ; \frac{-x}{(1-y)(1-z)}\right)\right]
\end{aligned}
$$$$
-(1+y)^{-a_{2}}(1-z)^{-a_{1}}
$$$$
\cdot\left[F\left(a_{1}, a_{2} ; c_{1} ; \frac{x}{(1+y)(1-z)}\right)+F\left(a_{1}, a_{2} ; c_{1} ; \frac{-x}{(1+y)(1-z)}\right)\right]
$$$$
+(1-y)^{-a_{2}}(1+z)^{-a_{1}}
$$$$
\cdot\left[F\left(a_{1}, a_{2} ; c_{1} ; \frac{x}{(1-y)(1+z)}\right)+F\left(a_{1}, a_{2} ; c_{1} ; \frac{-x}{(1-y)(1+z)}\right)\right]
$$ 


$$
\begin{aligned}
& -(1+y)^{-a_{2}}(1+z)^{-a_{1}} \\
& \cdot\left[F\left(a_{1}, a_{2} ; c_{1} ; \frac{x}{(1+y)(1+z)}\right)+F\left(a_{1}, a_{2} ; c_{1} ; \frac{-x}{(1+y)(1+z)}\right)\right] \text {; } \\
& 8 a_{2} y F^{(3)}\left[\begin{array}{rrrrr}
-:: & \frac{a_{2}+1}{2}, \frac{a_{2}+2}{2} ; & -; & \frac{a_{1}}{2}, \frac{a_{1}+1}{2} ; \\
-:: & -; & -; & -;
\end{array}\right. \\
& \left.\frac{c_{1}}{2}, \frac{c_{1}+1}{2}, \frac{1}{2} ; \quad-\frac{3}{2} ; \quad-\frac{1}{2} ; x^{2}, y^{2}, z^{2}\right] \\
& =(1-y)^{-a_{2}}(1-z)^{-a_{1}} \\
& \cdot\left[F\left(a_{1}, a_{2} ; c_{1} ; \frac{x}{(1-y)(1-z)}\right)+F\left(a_{1}, a_{2} ; c_{1} ; \frac{-x}{(1-y)(1-z)}\right)\right] \\
& -(1+y)^{-a_{2}}(1-z)^{-a_{1}} \\
& \cdot\left[F\left(a_{1}, a_{2} ; c_{1} ; \frac{x}{(1+y)(1-z)}\right)+F\left(a_{1}, a_{2} ; c_{1} ; \frac{-x}{(1+y)(1-z)}\right)\right] \\
& +(1-y)^{-a_{2}}(1+z)^{-a_{1}} \\
& \cdot\left[F\left(a_{1}, a_{2} ; c_{1} ; \frac{x}{(1-y)(1+z)}\right)+F\left(a_{1}, a_{2} ; c_{1} ; \frac{-x}{(1-y)(1+z)}\right)\right] \\
& -(1+y)^{-a_{2}}(1+z)^{-a_{1}} \\
& \text {. }\left[F\left(a_{1}, a_{2} ; c_{1} ; \frac{x}{(1+y)(1+z)}\right)+F\left(a_{1}, a_{2} ; c_{1} ; \frac{-x}{(1+y)(1+z)}\right)\right] \text {; }
\end{aligned}
$$

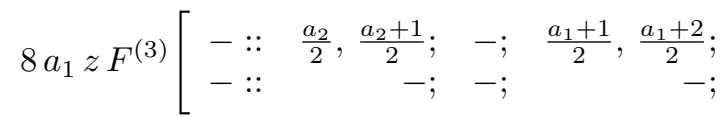

$$
\begin{aligned}
& \left.\frac{c_{1}}{2}, \frac{c_{1}+1}{2}, \frac{-1}{2} ; \quad-\frac{1}{2} ; \quad \frac{3}{2} ; x^{2}, y^{2}, z^{2}\right]
\end{aligned}
$$

$$
\begin{aligned}
= & (1-y)^{-a_{2}}(1-z)^{-a_{1}} \\
& \cdot\left[F\left(a_{1}, a_{2} ; c_{1} ; \frac{x}{(1-y)(1-z)}\right)+F\left(a_{1}, a_{2} ; c_{1} ; \frac{-x}{(1-y)(1-z)}\right)\right] \\
+ & (1+y)^{-a_{2}}(1-z)^{-a_{1}} \\
& \cdot\left[F\left(a_{1}, a_{2} ; c_{1} ; \frac{x}{(1+y)(1-z)}\right)+F\left(a_{1}, a_{2} ; c_{1} ; \frac{-x}{(1+y)(1-z)}\right)\right] \\
- & (1-y)^{-a_{2}}(1+z)^{-a_{1}} \\
& \cdot\left[F\left(a_{1}, a_{2} ; c_{1} ; \frac{x}{(1-y)(1+z)}\right)+F\left(a_{1}, a_{2} ; c_{1} ; \frac{-x}{(1-y)(1+z)}\right)\right]
\end{aligned}
$$




$$
\begin{aligned}
& -(1+y)^{-a_{2}}(1+z)^{-a_{1}} \\
& \text {. }\left[F\left(a_{1}, a_{2} ; c_{1} ; \frac{x}{(1+y)(1+z)}\right)+F\left(a_{1}, a_{2} ; c_{1} ; \frac{-x}{(1+y)(1+z)}\right)\right] \text {; }
\end{aligned}
$$

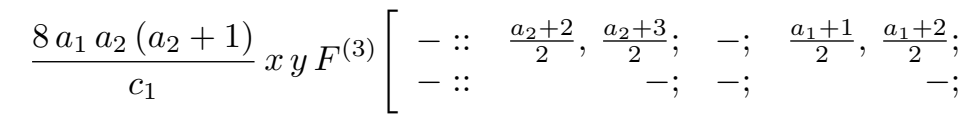

$$
\begin{aligned}
& \left.\frac{c_{1}+1}{2}, \frac{c_{1}+2}{2}, \frac{3}{2} ; \quad \frac{-3}{2} ; \quad \frac{-1}{2} ; x^{2}, y^{2}, z^{2}\right] \\
& =(1-y)^{-a_{2}}(1-z)^{-a_{1}} \\
& \text {. }\left[F\left(a_{1}, a_{2} ; c_{1} ; \frac{x}{(1-y)(1-z)}\right)-F\left(a_{1}, a_{2} ; c_{1} ; \frac{-x}{(1-y)(1-z)}\right)\right] \\
& -(1+y)^{-a_{2}}(1-z)^{-a_{1}} \\
& \text {. }\left[F\left(a_{1}, a_{2} ; c_{1} ; \frac{x}{(1+y)(1-z)}\right)-F\left(a_{1}, a_{2} ; c_{1} ; \frac{-x}{(1+y)(1-z)}\right)\right] \\
& +(1-y)^{-a_{2}}(1+z)^{-a_{1}} \\
& \text {. }\left[F\left(a_{1}, a_{2} ; c_{1} ; \frac{x}{(1-y)(1+z)}\right)-F\left(a_{1}, a_{2} ; c_{1} ; \frac{-x}{(1-y)(1+z)}\right)\right] \\
& -(1+y)^{-a_{2}}(1+z)^{-a_{1}} \\
& \text {. }\left[F\left(a_{1}, a_{2} ; c_{1} ; \frac{x}{(1+y)(1+z)}\right)-F\left(a_{1}, a_{2} ; c_{1} ; \frac{-x}{(1+y)(1+z)}\right)\right] \text {; }
\end{aligned}
$$

$$
\begin{aligned}
& \frac{8 a_{1}\left(a_{1}+1\right) a_{2}}{c_{1}} x z F^{(3)}\left[\begin{array}{rrrr}
-:: & \frac{a_{2}+1}{2}, \frac{a_{2}+2}{2} ; & -; & \frac{a_{1}+2}{2}, \frac{a_{1}+3}{2} ; \\
-:: & -; & -; &
\end{array}\right. \\
& \left.\frac{c_{1}+1}{2}, \frac{c_{1}+2}{2}, \frac{3}{2} ; \quad \frac{-1}{2} ; \quad \frac{-3}{2} ; x^{2}, y^{2}, z^{2}\right] \\
& =(1-y)^{-a_{2}}(1-z)^{-a_{1}} \\
& \text {. }\left[F\left(a_{1}, a_{2} ; c_{1} ; \frac{x}{(1-y)(1-z)}\right)-F\left(a_{1}, a_{2} ; c_{1} ; \frac{-x}{(1-y)(1-z)}\right)\right] \\
& +(1+y)^{-a_{2}}(1-z)^{-a_{1}} \\
& \text {. }\left[F\left(a_{1}, a_{2} ; c_{1} ; \frac{x}{(1+y)(1-z)}\right)-F\left(a_{1}, a_{2} ; c_{1} ; \frac{-x}{(1+y)(1-z)}\right)\right] \\
& -(1-y)^{-a_{2}}(1+z)^{-a_{1}} \\
& \text {. }\left[F\left(a_{1}, a_{2} ; c_{1} ; \frac{x}{(1-y)(1+z)}\right)-F\left(a_{1}, a_{2} ; c_{1} ; \frac{-x}{(1-y)(1+z)}\right)\right]
\end{aligned}
$$




$$
\begin{aligned}
& -(1+y)^{-a_{2}}(1+z)^{-a_{1}} \\
& \text {. }\left[F\left(a_{1}, a_{2} ; c_{1} ; \frac{x}{(1+y)(1+z)}\right)-F\left(a_{1}, a_{2} ; c_{1} ; \frac{-x}{(1+y)(1+z)}\right)\right] \text {; } \\
& 8 a_{1} a_{2} y z F^{(3)}\left[\begin{array}{rrrr}
-:: & \frac{a_{2}+1}{2}, \frac{a_{2}+2}{2} ; & -; & \frac{a_{1}+1}{2}, \frac{a_{1}+2}{2} ; \\
-:: & -; & -; & -;
\end{array}\right. \\
& \left.\frac{c_{1}}{2}, \frac{c_{1}+1}{2}, \frac{1}{2} ; \quad-\frac{3}{2} ; \quad-\frac{3}{2} ; x^{2}, y^{2}, z^{2}\right] \\
& =(1-y)^{-a_{2}}(1-z)^{-a_{1}} \\
& \cdot\left[F\left(a_{1}, a_{2} ; c_{1} ; \frac{x}{(1-y)(1-z)}\right)+F\left(a_{1}, a_{2} ; c_{1} ; \frac{-x}{(1-y)(1-z)}\right)\right] \\
& -(1+y)^{-a_{2}}(1-z)^{-a_{1}} \\
& \cdot\left[F\left(a_{1}, a_{2} ; c_{1} ; \frac{x}{(1+y)(1-z)}\right)+F\left(a_{1}, a_{2} ; c_{1} ; \frac{-x}{(1+y)(1-z)}\right)\right] \\
& -(1-y)^{-a_{2}}(1+z)^{-a_{1}} \\
& \cdot\left[F\left(a_{1}, a_{2} ; c_{1} ; \frac{x}{(1-y)(1+z)}\right)+F\left(a_{1}, a_{2} ; c_{1} ; \frac{-x}{(1-y)(1+z)}\right)\right] \\
& +(1+y)^{-a_{2}}(1+z)^{-a_{1}} \\
& \cdot\left[F\left(a_{1}, a_{2} ; c_{1} ; \frac{x}{(1+y)(1+z)}\right)+F\left(a_{1}, a_{2} ; c_{1} ; \frac{-x}{(1+y)(1+z)}\right)\right] \text {; }
\end{aligned}
$$

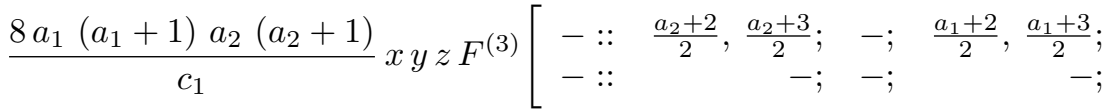

$$
\begin{aligned}
& \left.\frac{c_{1}+1}{2}, \frac{c_{1}+2}{2}, \frac{3}{2} ; \quad-\frac{3}{2} ; \quad-\frac{3}{2} ; x^{2}, y^{2}, z^{2}\right] \\
& =(1-y)^{-a_{2}}(1-z)^{-a_{1}} \\
& \cdot\left[F\left(a_{1}, a_{2} ; c_{1} ; \frac{x}{(1-y)(1-z)}\right)-F\left(a_{1}, a_{2} ; c_{1} ; \frac{-x}{(1-y)(1-z)}\right)\right] \\
& -(1-y)^{-a_{2}}(1+z)^{-a_{1}} \\
& \cdot\left[F\left(a_{1}, a_{2} ; c_{1} ; \frac{x}{(1-y)(1+z)}\right)-F\left(a_{1}, a_{2} ; c_{1} ; \frac{-x}{(1-y)(1+z)}\right)\right] \\
& -(1+y)^{-a_{2}}(1-z)^{-a_{1}} \\
& \cdot\left[F\left(a_{1}, a_{2} ; c_{1} ; \frac{x}{(1+y)(1-z)}\right)-F\left(a_{1}, a_{2} ; c_{1} ; \frac{-x}{(1+y)(1-z)}\right)\right]
\end{aligned}
$$




$$
\begin{aligned}
& +(1+y)^{-a_{2}}(1+z)^{-a_{1}} \\
& \quad \cdot\left[F\left(a_{1}, a_{2} ; c_{1} ; \frac{x}{(1+y)(1+z)}\right)-F\left(a_{1}, a_{2} ; c_{1} ; \frac{-x}{(1+y)(1+z)}\right)\right] .
\end{aligned}
$$

Similarly, setting $a_{3}=c_{2}$ in formulas (3.15) and (3.16) to (3.23), we find

Corollary 5. Each of the following relationships holds true.

$$
\begin{gathered}
{ }_{0} F_{1}\left(c_{1} ; x\right) e^{y+z} \\
=\left[{ }_{0} F_{3}\left(\frac{c_{1}}{2}, \frac{c_{1}+1}{2}, \frac{1}{2} ; \frac{x^{2}}{16}\right)+\frac{x}{c_{1}}{ }_{0} F_{3}\left(\frac{c_{1}+1}{2}, \frac{c_{1}+2}{2}, \frac{3}{2} ; \frac{x^{2}}{16}\right)\right] \\
\cdot\left[{ }_{0} F_{1}\left(\frac{1}{2} ; \frac{y^{2}}{4}\right)+y_{0} F_{1}\left(\frac{3}{2} ; \frac{y^{2}}{4}\right)\right]\left[{ }_{0} F_{1}\left(\frac{1}{2} ; \frac{z^{2}}{4}\right)+z_{0} F_{1}\left(\frac{3}{2} ; \frac{z^{2}}{4}\right)\right] ; \\
8_{0} F_{3}\left(\frac{c_{1}}{2}, \frac{c_{1}+1}{2}, \frac{1}{2} ; \frac{x^{2}}{16}\right){ }_{0} F_{1}\left(\frac{1}{2} ; \frac{y^{2}}{4}\right){ }_{0} F_{1}\left(\frac{1}{2} ; \frac{z^{2}}{4}\right) \\
=\left[{ }_{0} F_{1}\left(c_{1} ; x\right)+{ }_{0} F_{1}\left(c_{1} ;-x\right)\right]\left(e^{y}+e^{-y}\right)\left(e^{z}+e^{-z}\right) ;
\end{gathered}
$$

$$
\begin{aligned}
& \frac{8 x}{c_{1}}{ }_{0} F_{3}\left(\frac{c_{1}+1}{2}, \frac{c_{1}+2}{2}, \frac{3}{2} ; \frac{x^{2}}{16}\right){ }_{0} F_{1}\left(\frac{1}{2} ; \frac{y^{2}}{4}\right){ }_{0} F_{1}\left(\frac{1}{2} ; \frac{z^{2}}{4}\right) \\
& =\left[{ }_{0} F_{1}\left(c_{1} ; x\right)-{ }_{0} F_{1}\left(c_{1} ;-x\right)\right]\left(e^{y}+e^{-y}\right)\left(e^{z}+e^{-z}\right) \text {; } \\
& 8 y_{0} F_{3}\left(\frac{c_{1}}{2}, \frac{c_{1}+1}{2}, \frac{1}{2} ; \frac{x^{2}}{16}\right){ }_{0} F_{1}\left(\frac{3}{2} ; \frac{y^{2}}{4}\right){ }_{0} F_{1}\left(\frac{1}{2} ; \frac{z^{2}}{4}\right) \\
& =\left[{ }_{0} F_{1}\left(c_{1} ; x\right)+{ }_{0} F_{1}\left(c_{1} ;-x\right)\right]\left(e^{y}-e^{-y}\right)\left(e^{z}+e^{-z}\right) \text {; } \\
& 8 z_{0} F_{3}\left(\frac{c_{1}}{2}, \frac{c_{1}+1}{2}, \frac{1}{2} ; \frac{x^{2}}{16}\right){ }_{0} F_{1}\left(\frac{1}{2} ; \frac{y^{2}}{4}\right){ }_{0} F_{1}\left(\frac{3}{2} ; \frac{z^{2}}{4}\right) \\
& =\left[{ }_{0} F_{1}\left(c_{1} ; x\right)+{ }_{0} F_{1}\left(c_{1} ;-x\right)\right]\left(e^{y}+e^{-y}\right)\left(e^{z}-e^{-z}\right) \text {; } \\
& \frac{8 x y}{c_{1}}{ }_{0} F_{3}\left(\frac{c_{1}+1}{2}, \frac{c_{1}+2}{2}, \frac{3}{2} ; \frac{x^{2}}{16}\right){ }_{0} F_{1}\left(\frac{3}{2} ; \frac{y^{2}}{4}\right){ }_{0} F_{1}\left(\frac{1}{2} ; \frac{z^{2}}{4}\right) \\
& =\left[{ }_{0} F_{1}\left(c_{1} ; x\right)-{ }_{0} F_{1}\left(c_{1} ;-x\right)\right]\left(e^{y}-e^{-y}\right)\left(e^{z}+e^{-z}\right) \text {; } \\
& \frac{8 x z}{c_{1}}{ }_{0} F_{3}\left(\frac{c_{1}+1}{2}, \frac{c_{1}+2}{2}, \frac{3}{2} ; \frac{x^{2}}{16}\right){ }_{0} F_{1}\left(\frac{1}{2} ; \frac{y^{2}}{4}\right){ }_{0} F_{1}\left(\frac{3}{2} ; \frac{z^{2}}{4}\right) \\
& =\left[{ }_{0} F_{1}\left(c_{1} ; x\right)-{ }_{0} F_{1}\left(c_{1} ;-x\right)\right]\left(e^{y}+e^{-y}\right)\left(e^{z}-e^{-z}\right) \text {; }
\end{aligned}
$$




$$
\begin{aligned}
& 8 y z_{0} F_{3}\left(\frac{c_{1}}{2}, \frac{c_{1}+1}{2}, \frac{1}{2} ; \frac{x^{2}}{16}\right){ }_{0} F_{1}\left(\frac{3}{2} ; \frac{y^{2}}{4}\right){ }_{0} F_{1}\left(\frac{3}{2} ; \frac{z^{2}}{4}\right) \\
& \left.={ }_{0} F_{1}\left(c_{1} ; x\right)+{ }_{0} F_{1}\left(c_{1} ;-x\right)\right]\left(e^{y}-e^{-y}\right)\left(e^{z}-e^{-z}\right) ; \\
& \frac{8 x y z}{c_{1}}{ }_{0} F_{3}\left(\frac{c_{1}+1}{2}, \frac{c_{1}+2}{2}, \frac{3}{2} ; \frac{x^{2}}{16}\right){ }_{0} F_{1}\left(\frac{3}{2} ; \frac{y^{2}}{4}\right){ }_{0} F_{1}\left(\frac{3}{2} ; \frac{z^{2}}{4}\right) \\
& =\left[{ }_{0} F_{1}\left(c_{1} ; x\right)-{ }_{0} F_{1}\left(c_{1} ;-x\right)\right]\left(e^{y}-e^{-y}\right)\left(e^{z}-e^{-z}\right) ; \\
& \frac{x}{c_{1}} \frac{{ }_{0} F_{3}\left(\frac{c_{1}+1}{2}, \frac{c_{1}+2}{2}, \frac{3}{2} ; \frac{x^{2}}{16}\right)}{{ }_{0} F_{3}\left(\frac{c_{1}}{2}, \frac{c_{1}+1}{2}, \frac{1}{2} ; \frac{x^{2}}{16}\right)}=\frac{{ }_{0} F_{1}\left(c_{1} ; x\right)-{ }_{0} F_{1}\left(c_{1} ;-x\right)}{{ }_{0} F_{1}\left(c_{1} ; x\right)+{ }_{0} F_{1}\left(c_{1} ;-x\right)} ; \\
& y \frac{{ }_{0} F_{1}\left(\frac{3}{2} ; \frac{y^{2}}{4}\right)}{{ }_{0} F_{1}\left(\frac{1}{2} ; \frac{y^{2}}{4}\right)}=\frac{e^{y}-e^{-y}}{e^{y}+e^{-y} .}
\end{aligned}
$$

Setting $a_{2}=c_{1}$ in formulas (4.2) to (4.10), we can also express elementary power functions in terms of the Srivastava's function $F^{(3)}$ and vice-versa.

Corollary 6. Each of the following relationships holds true.

$$
\begin{aligned}
& (1-y)^{a_{1}-c_{1}}[(1-y)(1-z)-x]^{-a_{1}} \\
& =F^{(3)}\left[\begin{array}{rrrr}
-:: & \frac{c_{1}}{2}, \frac{c_{1}+1}{2} ; & -; & \frac{a_{1}}{2}, \frac{a_{1}+1}{2} ; \\
-:: & -; & -; & -;
\end{array}\right. \\
& \left.\frac{c_{1}}{2}, \frac{c_{1}+1}{2}, \frac{1}{2} ; \quad-\frac{1}{2} ; \quad-\frac{1}{2} ; x^{2}, y^{2}, z^{2}\right] \\
& +a_{1} x F^{(3)}\left[\begin{array}{rrrrr}
-:: & \frac{c_{1}+1}{2}, \frac{c_{1}+2}{2} ; & -; & \frac{a_{1}}{2}, \frac{a_{1}+1}{2} ; \\
-:: & -; & -; & -;
\end{array}\right. \\
& \left.\frac{c_{1}+1}{2}, \frac{c_{1}+2}{2}, \frac{-3}{2} ; \quad \frac{1}{2} ; \quad \frac{1}{2} ; x^{2}, y^{2}, z^{2}\right] \\
& +c_{1} y F^{(3)}\left[\begin{array}{rrrr}
-:: & \frac{c_{1}+1}{2}, \frac{c_{1}+2}{2} ; & -; & \frac{a_{1}}{2}, \frac{a_{1}+1}{2} ; \\
-:: & -; & -; & -;
\end{array}\right. \\
& \left.\frac{c_{1}}{2}, \frac{c_{1}+1}{2}, \frac{-1}{2} ; \quad \frac{3}{2} ; \quad \frac{-1}{2} ; x^{2}, y^{2}, z^{2}\right]
\end{aligned}
$$




$$
\begin{aligned}
& +a_{1} z F^{(3)}\left[\begin{array}{rrrr}
-:: & \frac{c_{1}}{2}, \frac{c_{1}+1}{2} ; & -; & \frac{a_{1}+1}{2}, \frac{a_{1}+2}{2} ; \\
-:: & -; & -; & -;
\end{array}\right. \\
& \left.\frac{c_{1}}{2}, \frac{c_{1}+1}{2}, \frac{-1}{2} ; \quad \frac{1}{2} ; \quad \frac{3}{2} ; x^{2}, y^{2}, z^{2}\right] \\
& +a_{1}\left(c_{1}+1\right) x y F^{(3)}\left[\begin{array}{rrrr}
-:: & \frac{c_{1}+2}{2}, \frac{c_{1}+3}{2} ; & -; & \frac{a_{1}+1}{2}, \frac{a_{1}+2}{2} ; \\
-:: & -; & -; & -;
\end{array}\right. \\
& \left.\frac{c_{1}+1}{2}, \frac{c_{1}+2}{2}, \frac{3}{2} ; \quad-\frac{3}{2} ; \quad-\frac{1}{2} ; x^{2}, y^{2}, z^{2}\right]
\end{aligned}
$$

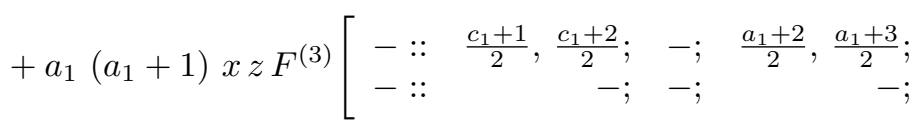

$$
\begin{aligned}
& \left.\frac{c_{1}+1}{2}, \frac{c_{1}+2}{2}, \frac{3}{2} ; \quad \frac{1}{2} ; \quad \frac{3}{2} ; x^{2}, y^{2}, z^{2}\right] \\
& +a_{1} c_{1} y z F^{(3)}\left[\begin{array}{rrrr}
-:: & \frac{c_{1}+1}{2}, \frac{c_{1}+2}{2} ; & -; & \frac{a_{1}+1}{2}, \frac{a_{1}+2}{2} ; \\
-:: & -; & -; & -;
\end{array}\right. \\
& \left.\frac{c_{1}}{2}, \frac{c_{1}+1}{2}, \frac{1}{2} ; \quad \frac{3}{2} ; \quad \frac{3}{2} ; x^{2}, y^{2}, z^{2}\right] \\
& +a_{1}\left(a_{1}+1\right)\left(c_{1}+1\right) x y z F^{(3)}\left[\begin{array}{rrrr}
-:: & \frac{c_{1}+2}{2}, \frac{c_{1}+3}{2} ; & -; & \frac{a_{1}+2}{2}, \frac{a_{1}+3}{2} ; \\
-:: & -; & -; & -;
\end{array}\right. \\
& \left.\frac{c_{1}+1}{2}, \frac{c_{1}+2}{2}, \frac{-3}{2} ; \quad \frac{3}{2} ; \quad \frac{3}{2} ; x^{2}, y^{2}, z^{2}\right] ;
\end{aligned}
$$

(4.23) $\quad 8 c_{1} y F^{(3)}\left[\begin{array}{rrrr}-:: & \frac{c_{1}}{2}, \frac{c_{1}+1}{2} ; & -; & \frac{a_{1}}{2}, \frac{a_{1}+1}{2} ; \\ -:: & -; & -; & -;\end{array}\right.$

$$
\begin{array}{r}
\left.\frac{c_{1}}{2}, \frac{c_{1}+1}{2}, \frac{1}{2} ; \quad \frac{1}{2} ; \quad \frac{1}{2} ; x^{2}, y^{2}, z^{2}\right] \\
=(1-y)^{a_{1}-c_{1}}\left\{[(1-y)(1-z)-x]^{-a_{1}}+[(1-y)(1-z)+x]^{-a_{1}}\right\} \\
+(1-y)^{a_{1}-c_{1}}\left\{[(1-y)(1+z)-x]^{-a_{1}}+[(1-y)(1+z)+x]^{-a_{1}}\right\} \\
+(1+y)^{a_{1}-c_{1}}\left\{[(1+y)(1-z)-x]^{-a_{1}}+[(1+y)(1-z)+x]^{-a_{1}}\right\} \\
+(1+y)^{a_{1}-c_{1}}\left\{[(1+y)(1+z)-x]^{-a_{1}}+[(1+y)(1+z)+x]^{-a_{1}}\right\} ;
\end{array}
$$




$$
\begin{aligned}
& 8 a_{1} x F^{(3)}\left[\begin{array}{rrrr}
-:: & \frac{c_{1}+1}{2}, \frac{c_{1}+2}{2} ; & -; & \frac{a_{1}}{2}, \frac{a_{1}+1}{2} ; \\
-:: & -; & -; & -;
\end{array}\right. \\
& \left.\frac{c_{1}+1}{2}, \frac{c_{1}+2}{2}, \frac{-3}{2} ; \quad-\frac{1}{2} ; \quad \frac{1}{2} ; x^{2}, y^{2}, z^{2}\right] \\
& =(1-y)^{a_{1}-c_{1}}\left\{[(1-y)(1-z)-x]^{-a_{1}}-[(1-y)(1-z)+x]^{-a_{1}}\right\} \\
& +(1+y)^{a_{1}-c_{1}}\left\{[(1+y)(1-z)-x]^{-a_{1}}-[(1+y)(1-z)+x]^{-a_{1}}\right\} \\
& +(1-y)^{a_{1}-c_{1}}\left\{[(1-y)(1+z)-x]^{-a_{1}}-[(1-y)(1+z)+x]^{-a_{1}}\right\} \\
& +(1+y)^{a_{1}-c_{1}}\left\{[(1+y)(1+z)-x]^{-a_{1}}-[(1+y)(1+z)+x]^{-a_{1}}\right\} ;
\end{aligned}
$$

$$
\begin{array}{r}
8 c_{1} y F^{(3)}\left[\begin{array}{rrrr}
-:: & \frac{c_{1}+1}{2}, \frac{c_{1}+2}{2} ; & -; & \frac{a_{1}}{2}, \frac{a_{1}+1}{2} ; \\
-:: & -; & -; & -; \\
\frac{c_{1}}{2}, \frac{c_{1}+1}{2}, \frac{1}{2} ; & \frac{3}{2} ; & \frac{1}{2} ;
\end{array} x^{2}, y^{2}, z^{2}\right] \\
=(1-y)^{a_{1}-c_{1}}\left\{[(1-y)(1-z)-x]^{-a_{1}}+[(1-y)(1-z)+x]^{-a_{1}}\right\} \\
-(1+y)^{a_{1}-c_{1}}\left\{[(1+y)(1-z)-x]^{-a_{1}}+[(1+y)(1-z)+x]^{-a_{1}}\right\} \\
+(1-y)^{a_{1}-c_{1}}\left\{[(1-y)(1+z)-x]^{-a_{1}}+[(1-y)(1+z)+x]^{-a_{1}}\right\} \\
-(1+y)^{a_{1}-c_{1}}\left\{[(1+y)(1+z)-x]^{-a_{1}}+[(1+y)(1+z)+x]^{-a_{1}}\right\}
\end{array}
$$

$$
\begin{aligned}
& 8 a_{1} z F^{(3)}\left[\begin{array}{rrrr}
-:: & \frac{c_{1}}{2}, \frac{c_{1}+1}{2} ; & -; & \frac{a_{1}+1}{2}, \frac{a_{1}+2}{2} ; \\
-:: & -; & -; & -;
\end{array}\right. \\
& \left.\frac{c_{1}}{2}, \frac{c_{1}+1}{2}, \frac{1}{2} ; \quad \frac{-1}{2} ; \quad \frac{-3}{2} ; x^{2}, y^{2}, z^{2}\right] \\
& =(1-y)^{a_{1}-c_{1}}\left\{[(1-y)(1-z)-x]^{-a_{1}}+[(1-y)(1-z)+x]^{-a_{1}}\right\} \\
& +(1+y)^{a_{1}-c_{1}}\left\{[(1+y)(1-z)-x]^{-a_{1}}+[(1+y)(1-z)+x]^{-a_{1}}\right\} \\
& -(1-y)^{a_{1}-c_{1}}\left\{[(1-y)(1+z)-x]^{-a_{1}}+[(1-y)(1+z)+x]^{-a_{1}}\right\} \\
& -(1+y)^{a_{1}-c_{1}}\left\{[(1+y)(1+z)-x]^{-a_{1}}+[(1+y)(1+z)+x]^{-a_{1}}\right\} \text {; }
\end{aligned}
$$


(4.27)

$$
\begin{aligned}
& 8 a_{1}\left(c_{1}+1\right) x y F^{(3)}\left[\begin{array}{rrrr}
-:: & \frac{c_{1}+2}{2}, \frac{c_{1}+3}{2} ; & -; & \frac{a_{1}+1}{2}, \frac{a_{1}+2}{2} ; \\
-:: & -; & -; & -;
\end{array}\right. \\
& \left.\frac{c_{1}+1}{2}, \frac{c_{1}+2}{2}, \frac{3}{2} ; \quad-\frac{3}{2} ; \quad-\frac{1}{2} ; x^{2}, y^{2}, z^{2}\right] \\
& =(1-y)^{a_{1}-c_{1}}\left\{[(1-y)(1-z)-x]^{-a_{1}}-[(1-y)(1-z)+x]^{-a_{1}}\right\} \\
& -(1+y)^{a_{1}-c_{1}}\left\{[(1+y)(1-z)-x]^{-a_{1}}-[(1+y)(1-z)+x]^{-a_{1}}\right\} \\
& +(1-y)^{a_{1}-c_{1}}\left\{[(1-y)(1+z)-x]^{-a_{1}}-[(1-y)(1+z)+x]^{-a_{1}}\right\} \\
& -(1+y)^{a_{1}-c_{1}}\left\{[(1+y)(1+z)-x]^{-a_{1}}-[(1+y)(1+z)+x]^{-a_{1}}\right\} \text {; }
\end{aligned}
$$

(4.28) $8 a_{1}\left(a_{1}+1\right) x z F^{(3)}\left[\begin{array}{rrrrr}-:: & \frac{c_{1}+1}{2}, \frac{c_{1}+2}{2} ; & -; & \frac{a_{1}+2}{2}, & \frac{a_{1}+3}{2} ; \\ -:: & -; & -; & -;\end{array}\right.$

$$
\begin{array}{r}
\left.\frac{c_{1}+1}{2}, \frac{c_{1}+2}{2}, \frac{3}{2} ; \quad \frac{1}{2} ; \quad \frac{3}{2} ; x^{2}, y^{2}, z^{2}\right] \\
=(1-y)^{a_{1}-c_{1}}\left\{[(1-y)(1-z)-x]^{-a_{1}}-[(1-y)(1-z)+x]^{-a_{1}}\right\} \\
+(1+y)^{a_{1}-c_{1}}\left\{[(1+y)(1-z)-x]^{-a_{1}}-[(1+y)(1-z)+x]^{-a_{1}}\right\} \\
-(1-y)^{a_{1}-c_{1}}\left\{[(1-y)(1+z)-x]^{-a_{1}}-[(1-y)(1+z)+x]^{-a_{1}}\right\} \\
-(1+y)^{a_{1}-c_{1}}\left\{[(1+y)(1+z)-x]^{-a_{1}}-[(1+y)(1+z)+x]^{-a_{1}}\right\} ;
\end{array}
$$

(4.29) $8 a_{1} c_{1} y z F^{(3)}\left[\begin{array}{rrrr}-:: & \frac{c_{1}+1}{2}, \frac{c_{1}+2}{2} ; & -; & \frac{a_{1}+1}{2}, \frac{a_{1}+2}{2} ; \\ -:: & -; & -; & -;\end{array}\right.$

$$
\begin{array}{r}
\left.\frac{c_{1}}{2}, \frac{c_{1}+1}{2}, \frac{1}{2} ; \quad \frac{3}{2} ; \quad \frac{3}{2} ; x^{2}, y^{2}, z^{2}\right] \\
=(1-y)^{a_{1}-c_{1}}\left\{[(1-y)(1-z)-x]^{-a_{1}}+[(1-y)(1-z)+x]^{-a_{1}}\right\} \\
-(1+y)^{a_{1}-c_{1}}\left\{[(1+y)(1-z)-x]^{-a_{1}}+[(1+y)(1-z)+x]^{-a_{1}}\right\} \\
-(1-y)^{a_{1}-c_{1}}\left\{[(1-y)(1+z)-x]^{-a_{1}}+[(1-y)(1+z)+x]^{-a_{1}}\right\} \\
+(1+y)^{a_{1}-c_{1}}\left\{[(1+y)(1+z)-x]^{-a_{1}}+[(1+y)(1+z)+x]^{-a_{1}}\right\} ;
\end{array}
$$




$$
\begin{aligned}
& 8 a_{1}\left(a_{1}+1\right)\left(c_{1}+1\right) x y z F^{(3)}\left[\begin{array}{rrrrr}
-:: & \frac{c_{1}+2}{2}, \frac{c_{1}+3}{2} ; & -; & \frac{a_{1}+2}{2}, & \frac{a_{1}+3}{2} ; \\
-:: & -; & -; &
\end{array}\right. \\
& \left.\frac{c_{1}+1}{2}, \frac{c_{1}+2}{2}, \frac{-3}{2} ; \quad-\frac{3}{2} ; \quad \frac{-3}{2} ; x^{2}, y^{2}, z^{2}\right] \\
& =(1-y)^{a_{1}-c_{1}}\left\{[(1-y)(1-z)-x]^{-a_{1}}-[(1-y)(1-z)+x]^{-a_{1}}\right\} \\
& -(1-y)^{a_{1}-c_{1}}\left\{[(1-y)(1+z)-x]^{-a_{1}}-[(1-y)(1+z)+x]^{-a_{1}}\right\} \\
& -(1+y)^{a_{1}-c_{1}}\left\{[(1+y)(1-z)-x]^{-a_{1}}-[(1+y)(1-z)+x]^{-a_{1}}\right\} \\
& +(1+y)^{a_{1}-c_{1}}\left\{[(1+y)(1+z)-x]^{-a_{1}}-[(1+y)(1+z)+x]^{-a_{1}}\right\} \text {. }
\end{aligned}
$$

\section{Concluding remarks}

We note that in a specialized parameters we can easily obtain many interesting functional relations from the identities established here. For instance, at $x=0$ and $z=0$ from (2.2) and (2.4) to (2.11) we can get decompositions for Appell's functions $F_{1}$ and $F_{2}$ in terms of Srivastava's function $F^{(3)}$.

Applying this method to some other special functions, instead of Srivastava's functions $H_{A}$ and $F^{(3)}$, defined by power series, interested readers can find certain other unexpected functional relations.

Acknowledgements This research was supported by Basic Science Research Program through the National Research Foundation of Korea funded by the Ministry of Education, Science and Technology(2012-0002957).

\section{References}

[1] M. Abramowitz and I. A. Stegun, Handbook of Mathematical Functions with Formulas, Graphs, and Mathematical Tables, Applied Mathematics Series, 55, National Bureau of Standards, Washington, D. C., 1964; Reprinted by Dover Publications, New York, 1965.

[2] A. Altin, Some expansion formulas for a class of singular partial differential equations, Proc. Amer. Math. Soc., 85(1)(1982), 42-46.

[3] P. Appell and J. Kampé de Fériet, Fonctions Hypergeometriques et Hyperspheriques; Polynomes d'Hermite, Gauthier - Villars, Paris, 1926.

[4] J. Barros-Neto and I. M. Gelfand, Fundamental solutions for the Tricomi operator, Duke Math. J., 98(3)(1999), 465-483.

[5] J. Barros-Neto and I. M. Gelfand, Fundamental solutions for the Tricomi operator II, Duke Math. J., 111(3)(2002), 561-584. 
[6] J. Barros-Neto and I. M. Gelfand, Fundamental solutions for the Tricomi operator III, Duke Math. J., 128(1)(2005), 119-140.

[7] L. Bers, Mathematical Aspects of Subsonic and Transonic Gas Dynamics, Wiley, New York, 1958.

[8] B. C. Carlson, Some extensions of Lardner's relations between ${ }_{0} F_{3}$ and Bessel functions, SIAM J. Math. Anal., 1(2)(1970), 232-242.

[9] A. Erdélyi, W. Magnus, F. Oberhettinger and F. G. Tricomi, Higher Transcendental Functions, Vol. 1, McGraw-Hill Book Company, New York, Toronto and London, 1953.

[10] A. Erdélyi, W. Magnus, F. Oberhettinger and F. G. Tricomi, Higher Transcendental Functions, Vol. 2, McGraw-Hill Book Company, New York, Toronto and London, 1953.

[11] F. I. Frankl, Selected Works in Gas Dynamics. Nauka, Moscow 1973.

[12] A. J. Fryant, Growth and complete sequences of generalized bi-axially symmetric potentials, J. Diff. Equa., 31(2)(1979), 155-164.

[13] A. Hasanov, Fundamental solutions of generalized bi-axially symmetric Helmholtz equation, Complex Variables and Elliptic Equations 52(8)(2007), 673-683.

[14] A. Hasanov, Some solutions of generalized Rassias's equation, Intern. J. Appl. Math. Stat., 8(M07)(2007), 20-30.

[15] A. Hasanov, Fundamental solutions for degenerated elliptic equation with two perpendicular lines of degeneration, Intern. J. Appl. Math. Stat., 13(8)(2008), 41-49.

[16] A. Hasanov and E. T. Karimov, Fundamental solutions for a class of threedimensional elliptic equations with singular coefficients, Appl. Math. Lett., 22(2009), 1828-1832.

[17] A. Hasanov, J. M. Rassias and M. Turaev, Fundamental solution for the generalized Elliptic Gellerstedt Equation, Book: Functional Equations, Difference Inequalities and ULAM Stability Notions, Nova Science Publishers Inc. NY, USA, 6(2010), 73-83.

[18] A. Hasanov and H. M. Srivastava, Some decomposition formulas associated with the Lauricella Function and other multiple hypergeometric functions, Appl. Math. Lett., 19(2006), 113-121.

[19] A. Hasanov and H. M. Srivastava, Decomposition formulas associated with the Lauricella multivariable hypergeometric functions, Comput. Math. Appl., 53(7)(2007), 1119-1128.

[20] A. Hasanov, H. M. Srivastava, and M. Turaev, Decomposition formulas for some triple hypergeometric functions, J. Math. Anal. Appl., 324(2006), 955-969.

[21] A. Hasanov and M. Turaev, Decomposition formulas for the double hypergeometric G1 and G2 Hypergeometric functions, Appl. Math. Comput., 187(1)(2007), 195-201.

[22] Y. S. Kim, A. K. Rathie and J. Choi, Note on Srivastava's triple hypergeometric series $H_{A}$, Commun. Korean Math. Soc., 18(3)(2003), 581-586.

[23] T. J. Lardner, Relations between ${ }_{0} F_{3}$ and Bessel functions, SIAM Review, 11(1969), 69-72. 
[24] T. J. Lardner and C. R. Steele, Symmetric deformations of circular cylindrical elastic shells of exponentially varying thickness, Trans. ASME Ser. E. J. Appl. Mech., 35(1968), 169-170.

[25] G. Lohofer, Theory of an electromagnetically deviated metal sphere. 1: Absorbed power. SIAM J. Appl. Math., 49(1989), 567-581.

[26] P. A. McCoy, Polynomial approximation and growth of generalized axisymmetric potentials, Canad. J. Math., 31(1)(1979), 49-59.

[27] A. W. Niukkanen, Generalized hypergeometric series arising in physical and quantum chemical applications, J. Phys. A: Math. Gen., 16(1983), 1813-1825.

[28] A. K. Rathie and Y. S. Kim, Further results on Srivastava's triple hypergeometric series $H_{A}$ and $H_{C}$, Ind. J. Pure Appl. Math., 35(8)(2004), 991-1002.

[29] M. S. Salakhitdinov and A. Hasanov, A solution of the Neumann-Dirichlet boundary value problem for generalized bi-axially symmetric Helmholtz equation, Complex Variables and Elliptic Equations, 53(4)(2008), 355-364.

[30] H. M. Srivastava, Hypergeometric functions of three variables, Ganita, 15(2)(1964), 97-108.

[31] H. M. Srivastava, Some integrals representing hypergeometric functions, Rend. Circ. Mat. Palermo, 16(2)(1967), 99-115.

[32] H. M. Srivastava and J. Choi, Series Associated with the Zeta and Related Functions, Kluwer Academic Publishers, Dordrecht, Boston and London, 2001.

[33] H. M. Srivastava and P. W. Karlsson, Multiple Gaussian Hypergeometric Series, Halsted Press(Ellis Horwood Limited, Chichester), Wiley, New York, Chichester, Brisbane, and Toronto, 1985.

[34] M. Turaev, Decomposition formulas for Srivastava's hypergeometric function on Saran functions, Comput. Appl. Math., 233(2009), 842-846.

[35] G. N. Watson, A Treatise on the Theory of Bessel Functions, 2nd Edi., Cambridge University Press, Cambridge, London and New York, 1944.

[36] A. Weinstein, Discontinuous integrals and generalized potential theory, Trans. Amer. Math. Soc., 63(1946), 342-354.

[37] A. Weinstein, Generalized axially symmetric potential theory, Bull. Amer. Math. Soc., 59(1953), 20-38.

[38] R. J. Weinacht, Fundamental solutions for a class of singular equations, Contrib. Diff. Equa., 3(1964), 43-55. 